\title{
Upper-Body Post-activation Performance Enhancement for Athletic Performance: A Systematic Review with Meta-analysis and Recommendations for Future Research
}

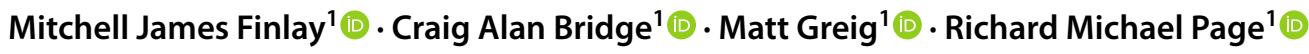

Accepted: 4 November 2021 / Published online: 26 November 2021

(c) The Author(s) 2021, corrected publication 2021

\begin{abstract}
Background Research on post-activation performance enhancement (PAPE) is dominated by lower-body conditioning activities/performance test complexes. Despite the contribution of the upper body to many sporting actions, no review on upperbody PAPE currently exists.

Objectives The aim of this systematic review with meta-analysis was to provide a synthesis of the available research on the inclusion of upper-body PAPE conditioning activities to improve athletic performance.

Methods A review of the literature was conducted according to the Preferred Reporting Items for Systematic Review and Meta-analyses guidelines, including a literature search of EBSCOhost, SPORTDiscus, PubMed and Google Scholar databases. A total of 127 studies were identified through database searches, and were assessed against the following criteria: (1) randomised controlled trial or pre-and-post study design; (2) studies explored the effects of prior voluntary muscle activity, and not electrically induced contractions, (3) evidence, or lack thereof, of PAPE was quantified by the monitoring of individual performance to commonly applied physical tests or sport-specific tasks; (4) conditioning activities and performance tests were primarily upper-body; (5) detailed description of a standardised warm-up; and (6) full-text versions of studies could be accessed in English language peer-reviewed journals. Studies were quality assessed for methodological quality via the PEDro scale and ranked accordingly.

Results Thirty-one studies met the inclusion criteria. Studies were classified into different conditioning activity modes: bench press variations, sport-specific (modified implement throws, swing-specific, cable pulley, elastic resistance, combination) and bodyweight activity. Acute performance enhancement in several movement-specific combinations was found. A metaanalysis revealed that bench press at $\geq 80 \%$ one repetition maximum significantly $(p=0.03$; ES $=0.31)$ improves subsequent power output in the ballistic bench throw at 30-40\% one repetition maximum, following 8-12 min recovery. Additionally, sport-specific overweight implement throws improved subsequent throwing distance at competition weight by 1.7-8.5\%; $\mathrm{ES}=0.14-0.33$, following 3 min recovery. Sport-specific lighter weighted bat swings and swing-specific isometrics resulted in improved subsequent competition weight bat swing velocities, ranging from $~ 1.3-4.9 \%$; ES $=0.16-0.57$.

Conclusions This review presents several upper-body movement-specific conditioning activities that could be considered by coaches and practitioners as part of complex or contrast training, or used in pre-competition warm-ups to acutely enhance performance.
\end{abstract}

\section{Introduction}

Coaches and practitioners regularly utilise the pre-competition warm-up to acutely enhance neuromuscular performance [1-3]. The use of a warm-up is thought to influence

Mitchell James Finlay

Finlaym@edgehill.ac.uk

Sports Injuries Research Group, Department of Sport and Physical Activity, Edge Hill University, St. Helens Road, Ormskirk L39 4QP, Lancashire, UK performance through several temperature-related (decreased resistance of muscles and joints, increased nerve conduction rate and thermoregulatory strain, greater release of oxygen from haemoglobin and myoglobin, and speeding up of metabolic reactions) and non-temperature-related (increased blood flow, elevation of baseline oxygen consumption and psychological effects) mechanisms [3]. Likewise, it is possible that the use of an additional conditioning activity may also influence subsequent neuromuscular performance [4], possibly above and beyond that of the warm-up [5]. An 


\section{Key Points}

Bench press of $\geq 80 \% 1 \mathrm{RM}$ induces a moderate postactivation performance enhancement effect in the subsequent ballistic bench throw.

Sport-specific post-activation performance enhancement conditioning activities, including overweight implement throw and lightweight and isometric bat swings, improved subsequent throwing distance and bat swing velocity, respectively.

Upper-body conditioning activities that share biomechanical specificity with the performance test may be more likely to induce a post-activation performance enhancement effect.

example use of a conditioning activity, is complex training, whereby the completion of a high-load resistance activity can enhance subsequent plyometric or ballistic-type activity $[4,6,7]$. The mechanisms and terminology of this acute exercise performance enhancement, or previously termed 'post-activation potentiation' (PAP), have recently been subject to debate within the scientific community, resulting in the term 'post-activation performance enhancement' (PAPE) being proposed [8]. Blazevich and Babault [5] suggest that PAPE may be attributable to mechanisms that are commonly observed through warm-ups, such as changes in muscle temperature and intramuscular fluid accumulation, or neural mechanisms. However, the specific mechanisms require further investigation. A delayed, yet prolonged window of action is associated with PAPE, and is often observable by improvements in neuromuscular performance lasting several minutes in responding athletes $[5,9,10]$. Where PAP may also occur and coexist with PAPE in response to prior voluntary action [9], it is typically dissipated in a manner of minutes, often by the time PAPE effects are evident [5]. With consideration to the traditional misuse of PAP, and the ongoing debates on revised nomenclature $[5,9,11,12]$, this current review considers studies that have assessed the efficacy of prior voluntary muscular activity, on the subsequent performance of a voluntary activity, as PAPE.

Considerable inter-individual variability exists in the PAPE response, from a positive effect (responders), no effect (non-responders) or even adverse effects [13]. There appear to be several modulating factors including, but not limited to, participant strength levels, sex, conditioning activity, type of load, warm-up activity, rest-period and performance test $[4,5]$. The warm-up is perhaps of increased importance, in that it could also explain any performance enhancement as opposed to, or in parallel with the conditioning activity, considering that the proposed mechanisms are similar. Nevertheless, recent reviews related to PAPE show that numerous forms of prior voluntary activity can improve subsequent neuromuscular performance, such as barbell compound lifts, plyometric, ballistic, variable resistance, resisted sprints and isometric activity $[4,14$, 15]. These reviews have predominantly focussed on lowerbody PAPE conditioning activities and performance tests $[4,7,14]$ because of a paucity of research pertaining to the upper-body, perhaps with the exception of bench press variations [14]. The recent increase in experimental research on upper-body PAPE means it is now conceivable to perform a more focused and up-to-date systematic review and metaanalysis in this area.

Seitz and Haff [4] reported small and moderate PAPE effects for jumping (effect size $[E S]=0.31$ ) and sprinting $(E S=0.50)$ performance, in addition to small effects for throwing $(E S=0.28)$ and upper-body ballistic performance $(\mathrm{ES}=0.23)$. Whilst this suggests upper-body performance can be aided by PAPE, important detail on the different types and characteristics of individual upper-body PAPE conditioning activities was often lacking. Furthermore, there may be a need for a more up-to-date review inclusive of more recent PAPE research in the literature. Considering the above reasons, a more comprehensive and recent review of literature on upper-body complexes is required.

Therefore, this study aims to conduct a systematic review on the current evidence of upper-body PAPE conditioning activities. To do this, a systematic process of literature searching, data extraction and quality assessment is conducted. Data from relevant studies are collated and shown as percentage changes and ES where possible. Finally, where appropriate, a meta-analysis of similar study designs will be performed. Considering the importance of upper-body strength and power to many sports [16], a more comprehensive and up-to-date review on upper-body PAPE could be valuable for the coach and practitioner in assisting the selection and use of acute strategies to facilitate performance for short-term, medium-term and long-term goals.

\section{Methods}

\subsection{Literature Search}

A review of the literature was conducted according to the Preferred Reporting Items for Systematic Review and Metaanalyses (PRISMA) guidelines [17] (Fig. 1). EBSCOhost, SPORTDiscus, PubMed and Google Scholar databases were searched until August 2021 for all studies pertaining to acute upper-body PAPE. Several combinations of search terms detailed in Table 1 were used. Reference lists of the identified manuscripts were searched manually. 


\subsection{Data Extraction}

The screening and data extraction process was carried out by two independent reviewers (MF and RP) and included study results, sample size, sex, experience level and strength levels. Any discrepancies between the two reviewers were discussed between the authors until a consensus was reached. Studies were assessed for methodological quality at the study level using the Physiotherapy Evidence Database (PEDro) scale [18]. Total PEDro scores are reached based on satisfaction of criterion measures relating to participant allocation, allocation concealment, blinding of participants, therapists and assessors, and the provision of sufficient statistical information [18]. A total of 11 criterion measures are assessed; however, criterion measures 1 and 6 are to assess external validity and blinding of therapists who administered the therapy, and are not included in the total PEDro score. Therefore, a total score of 9 is attainable.

\subsection{Study Identification and Selection}

For inclusion in the review, the following criteria were required to be satisfied: (1) randomised controlled trial or pre-and-post study design; (2) studies explored the effects of prior voluntary muscle activity; (3) evidence, or lack thereof, of PAPE was quantified by the monitoring of individual performance to commonly applied physical tests or sport-specific tasks; (4) conditioning activities and performance tests were primarily upper body; (5) studies included a detailed description of an appropriate, standardised warm-up; (6)
Fig. 1 Preferred Reporting Items for Systematic Reviews and Meta-Analysis (PRISMA) flow diagram of the literature screening process. $C A$ conditioning activity

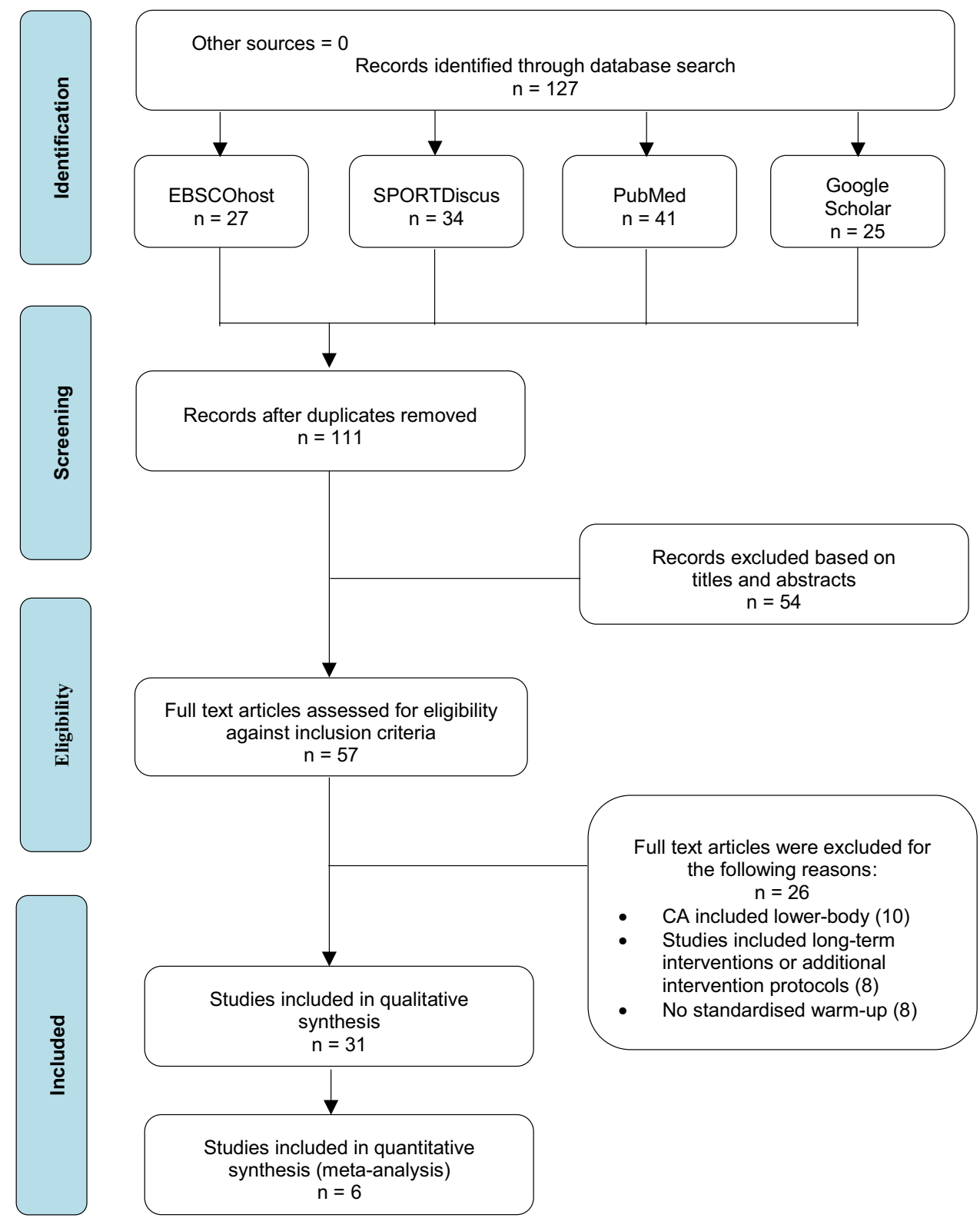


Table 1 Search criteria

Search terms

"Post-activation potentiation" OR "PAP" OR "post-activation performance enhancement" OR "PAPE" OR "warm-up" OR "contrast" OR

"complex" OR "acute" OR "acutely" OR "effects" OR "short-term" OR “preparedness" OR "pre-activation" OR "activation" OR "resisted

warm-up" OR "pre-competition” OR "neuromuscular" OR "strength” OR "power"

AND

"Upper-body” OR “throwing” OR "ballistic” OR “striking” OR "bench press" OR “dry-land” OR "exercise” OR "series" OR "bout” OR "athlete" OR "athletic" OR "sport"

full-text versions of studies could be accessed in English language, in peer-reviewed journals. Studies were excluded from the review if they included any of the following criteria: (1) a conditioning activity or performance measure that was primarily lower body, (2) long-term interventions or considered other interventions, such as nutritional supplementation; and (3) no details of a standardised warm-up.

\subsection{Coding of the Studies}

The same two authors that performed the data extraction (MF and RP) performed the study coding. Specifically, studies that passed eligibility criteria were classified into groups according to the conditioning activity performed, which included bench press variations, sport-specific, bodyweight and combined activity. The bench press variation category included studies that explored the bench press and eccentric, concentric, isometric and variable resistance variations of the bench press. The sport-specific category included studies that investigated the use of a conditioning activity that replicated the sporting task. This included modified implement throws, swing-specific activity in ball striking sports, cable pulley and elastic resistance conditioning activities. Studies that included only bodyweight activity, or included a combination of exercises, were classified as such.

\subsection{Risk of Bias Analysis}

Articles that passed the eligibility criteria were ranked on their methodological quality via the PEDro scale [18]. This comprised the binary scoring $(0 / 1)$ of whether articles followed 9/11 discrete criteria, Table 2. Criterion 1 was concerned with assessing external validity; therefore, it was deemed not applicable for this review. Similarly, criterion 6 was omitted. Studies were subsequently scored on the remaining nine criteria.

\subsection{Reporting of Results}

Results from the included studies are presented in the main text and in Table 3 as percentage changes. Effect sizes were also calculated, according to a previous review relating to PAPE [4] and more recently [19]. Specifically, the ES were calculated using Hedges and Olkin's $g$ (Hedges $g$ ) [20] as follows (Eq. 1):

$\mathrm{ES}=\mathrm{g} \frac{\left(M_{\text {post }}-M_{\text {pre }}\right)}{\mathrm{SD}_{\text {pooled }}}$,

where $M_{\text {post }}$ is the mean of the performance test completed after the conditioning activity, $M_{\text {pre }}$ is the mean of the performance test completed before the CA and $S D_{\text {pooled }}$ is the pooled standard deviation of the measurements (Eq. 2):

$\mathrm{SD}_{\text {pooled }}=\frac{\left(\left(\mathrm{n}_{1}-1\right) \times \mathrm{SD}_{1}^{2}+\left(\mathrm{n}_{2}-1\right) \times \mathrm{SD}_{2}^{2}\right)}{\left(\mathrm{n}_{1}+\mathrm{n}_{2}-2\right)}$,

where $\mathrm{SD}_{1}^{2}$ is the standard deviation of the performance test completed before the $\mathrm{CA}$ and $\mathrm{SD}_{2}^{2}$ is the standard deviation of the performance test completed after the conditioning activity. Hedges and Olkin [20] suggest the absolute value of the ES is over-estimated where there are small sample sizes. The authors advise that the ES should be corrected (Hedges $g^{*}$ ) as using the following equation (Eq. 3):

Correction factor $=1-\frac{3}{4\left(n_{1}+n_{2}-2\right)-1}$.

Seitz and Haff [4] note that this method is preferable in pre-test and post-test design studies in meta-analyses, citing Morris [21] who suggested that this method shows superior properties with respect to bias, precision, and robustness to heterogeneity of variance compared with other methods. The corrected ES was then calculated as follows (Eq. 4):

Corrected ES $=g \times$ correction factor

The following thresholds were used: trivial $=0.20$, small $=0.20-0.49$, moderate $=0.50-0.80$, and large $=\geq 0.80$ [22].

\subsection{Meta-analysis}

Where appropriate, a meta-analysis was performed using RevMan, version 5.4 [23]. The standardised mean difference of pre-post changes was calculated, and heterogeneity between studies was assessed by observing the $I^{2}$ statistic, at the following thresholds: $0-40 \%$ may not be important, 
Table 2 PEDro scale quality assessment of the articles [21]

\begin{tabular}{|c|c|c|c|c|c|c|c|c|c|c|c|c|}
\hline Reference & $1^{\mathrm{a}}$ & 2 & 3 & 4 & 5 & $6^{\mathrm{a}}$ & 7 & 8 & 9 & 10 & 11 & Total \\
\hline Ulrich and Parstorfer [25] & - & 1 & 0 & 0 & 0 & - & 0 & 1 & 1 & 1 & 1 & $5 / 9$ \\
\hline Judge et al. [26] & - & 1 & 0 & 0 & 0 & - & 0 & 1 & 1 & 1 & 1 & $5 / 9$ \\
\hline West et al. [27] & - & 0 & 0 & 0 & 0 & - & 0 & 1 & 1 & 1 & 1 & $4 / 9$ \\
\hline Tsolakis et al. [28] & - & 1 & 0 & 1 & 0 & - & 0 & 1 & 1 & 1 & 1 & $6 / 9$ \\
\hline Ferreira et al. [29] & - & 1 & 0 & 0 & 0 & - & 0 & 1 & 1 & 1 & 1 & $5 / 9$ \\
\hline Abbes et al. [30] & - & 1 & 0 & 1 & 0 & - & 0 & 1 & 1 & 1 & 1 & $6 / 9$ \\
\hline Krzysztofik [31] & - & 1 & 0 & 1 & 0 & - & 0 & 1 & 1 & 1 & 1 & $6 / 9$ \\
\hline Krzysztofik and Wilk [32] & - & 1 & 0 & 1 & 0 & - & 0 & 1 & 1 & 1 & 1 & $6 / 9$ \\
\hline Markovic et al. [33] & - & 1 & 0 & 1 & 0 & - & 0 & 1 & 1 & 1 & 1 & $6 / 9$ \\
\hline Brandenburg [34] & - & 1 & 0 & 1 & 0 & - & 0 & 1 & 1 & 1 & 1 & $6 / 9$ \\
\hline Esformes et al. [35] & - & 1 & 0 & 1 & 0 & - & 0 & 1 & 1 & 1 & 1 & $6 / 9$ \\
\hline Montoya et al. [36] & - & 1 & 0 & 0 & 0 & - & 0 & 1 & 1 & 1 & 1 & $5 / 9$ \\
\hline Bellar et al. [37] & - & 1 & 0 & 0 & 0 & - & 0 & 1 & 1 & 1 & 1 & $5 / 9$ \\
\hline Bevan et al. [38] & - & 0 & 0 & 0 & 0 & - & 0 & 1 & 1 & 1 & 1 & $4 / 9$ \\
\hline Bodden et al. [39] & - & 1 & 0 & 1 & 0 & - & 0 & 1 & 1 & 1 & 1 & $6 / 9$ \\
\hline Kilduff et al. [40] & - & 0 & 0 & 0 & 0 & - & 0 & 1 & 1 & 1 & 1 & $4 / 9$ \\
\hline Liossis et al. [41] & - & 1 & 0 & 0 & 0 & - & 0 & 1 & 1 & 1 & 1 & $5 / 9$ \\
\hline Judge et al. [42] & - & 1 & 0 & 0 & 0 & - & 0 & 1 & 1 & 1 & 1 & $5 / 9$ \\
\hline Gilmore et al. [43] & - & 0 & 0 & 0 & 0 & - & 0 & 1 & 1 & 1 & 1 & $4 / 9$ \\
\hline Hancock et al. [44] & - & 1 & 0 & 1 & 0 & - & 0 & 1 & 1 & 1 & 1 & $6 / 9$ \\
\hline Barbosa et al. [45] & - & 1 & 0 & 1 & 0 & - & 0 & 1 & 1 & 1 & 1 & $6 / 9$ \\
\hline Martinez-Garcia et al. [46] & - & 0 & 0 & 1 & 0 & - & 0 & 1 & 1 & 1 & 1 & $5 / 9$ \\
\hline Gelen et al. [47] & - & 1 & 0 & 1 & 0 & - & 0 & 1 & 1 & 1 & 1 & $6 / 9$ \\
\hline Cuenca-Fernandez et al. [48] & - & 1 & 0 & 1 & 0 & - & 0 & 1 & 1 & 1 & 1 & $6 / 9$ \\
\hline Bliss et al. [49] & - & 0 & 0 & 1 & 0 & - & 0 & 1 & 1 & 1 & 1 & $5 / 9$ \\
\hline Feros. $2020[50]$ & - & 1 & 0 & 0 & 0 & - & 0 & 1 & 1 & 1 & 1 & $5 / 9$ \\
\hline Higuchi et al. [51] & - & 0 & 0 & 1 & 0 & - & 0 & 1 & 1 & 1 & 1 & $5 / 9$ \\
\hline Williams et al. [52] & - & 1 & 0 & 1 & 0 & - & 0 & 1 & 1 & 1 & 1 & $6 / 9$ \\
\hline Sarramian et al. [53] & - & 0 & 0 & 0 & 0 & - & 0 & 1 & 1 & 1 & 1 & $4 / 9$ \\
\hline Smilios et al. [54] & - & 1 & 0 & 1 & 0 & - & 0 & 1 & 1 & 1 & 1 & $6 / 9$ \\
\hline Asencio et al. [55] & - & 1 & 0 & 0 & 0 & - & 0 & 1 & 1 & 1 & 1 & $5 / 9$ \\
\hline
\end{tabular}

$1=$ criterion was satisfied, $0=$ criterion was not satisfied. Each satisfied criterion measure, excluding item 1 and 6, contributes 1 point to the total PEDro score (1-9). Criteria: (1) eligibility criteria were specified ('anot applicable); (2) random allocation; (3) concealed allocation; (4) groups similar at baseline; (5) blinding of participants; (6) blinding of therapists who administered the therapy ( ${ }^{a}$ not applicable); (7) blinding of assessors; (8) less than 15\% drop-outs; (9) intention to treat; (10) between-group statistical analysis; (11) point measures and variability data

PEDro Physiotherapy Evidence Database
30-60\% may represent moderate heterogeneity, 50-90\% may represent substantial heterogeneity and $\geq 75 \%$ may represent considerable heterogeneity [24].

\section{Results}

A total of 127 studies pertaining to the search criteria were identified for further analysis, of which 16 duplicates were removed. Titles and abstracts of the 111 manuscripts were initially screened for their relevance, followed by a full-text review of the remaining 57 relevant manuscripts to assess their eligibility in accordance with the inclusion criteria. After analysing eligibility, 31 studies were selected to be included in the review (Fig. 1).

\subsection{Risk of Bias Analysis}

As identified in Table 2, the methodological quality of 26 studies was $\geq 5 / 9$, whilst five studies were classed as $4 / 9$, which translated to median score of 5 . No studies were identified as $<4$. Criteria 1 and 6 were omitted because of a lack of relevance. Minimal information for criteria 5, 6 and 7 was evident because of the nature of the experimental studies. 


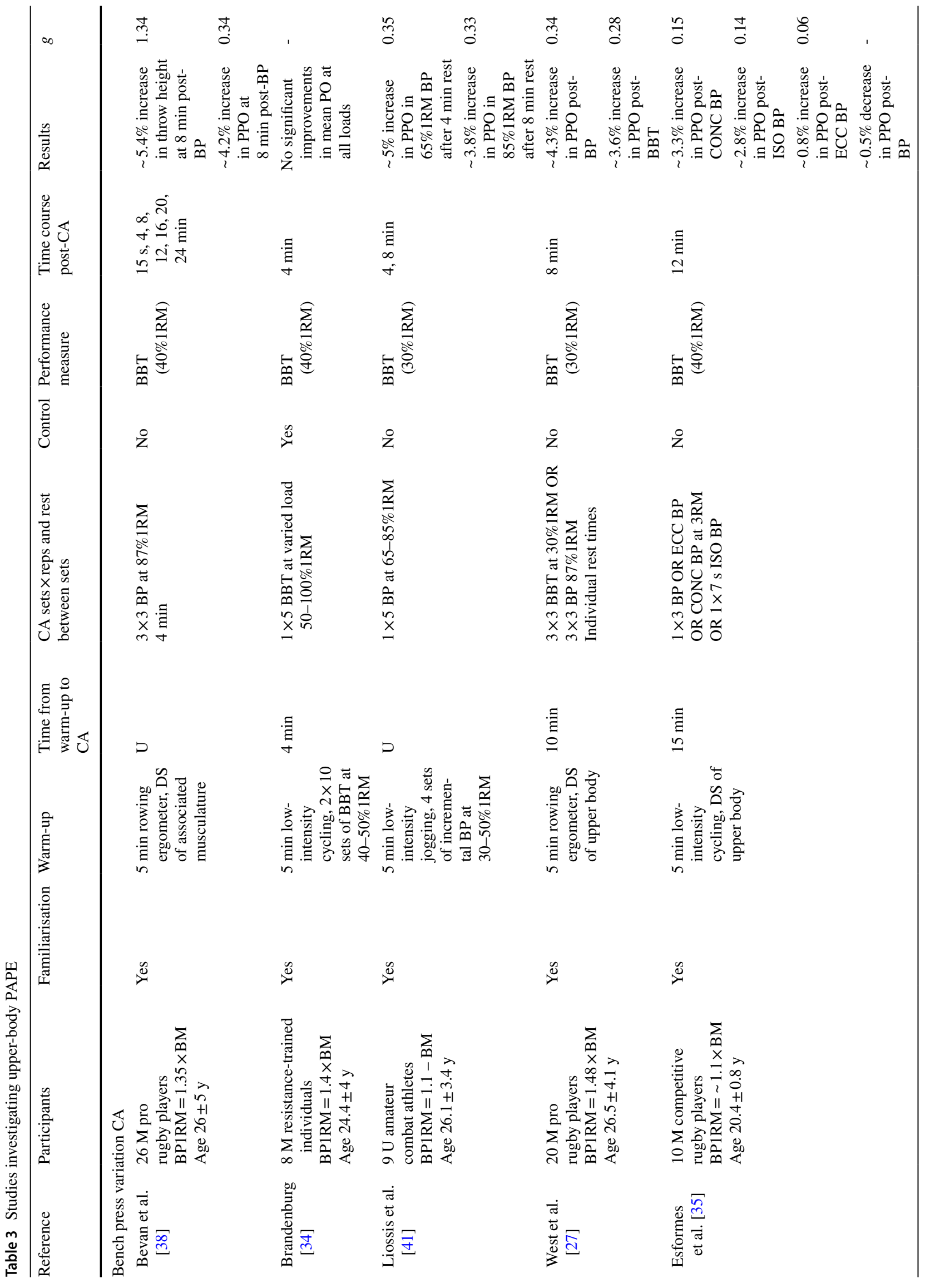




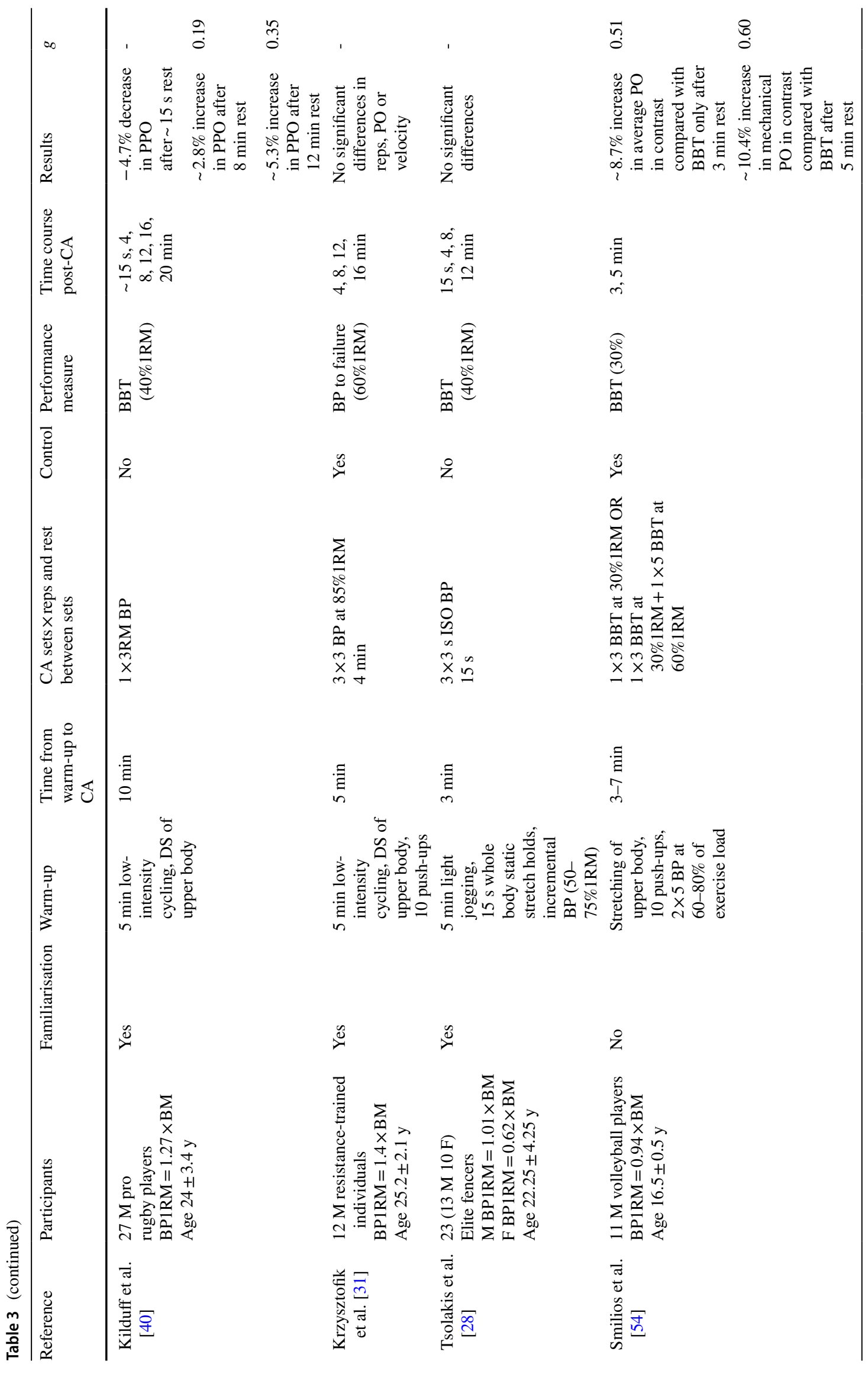




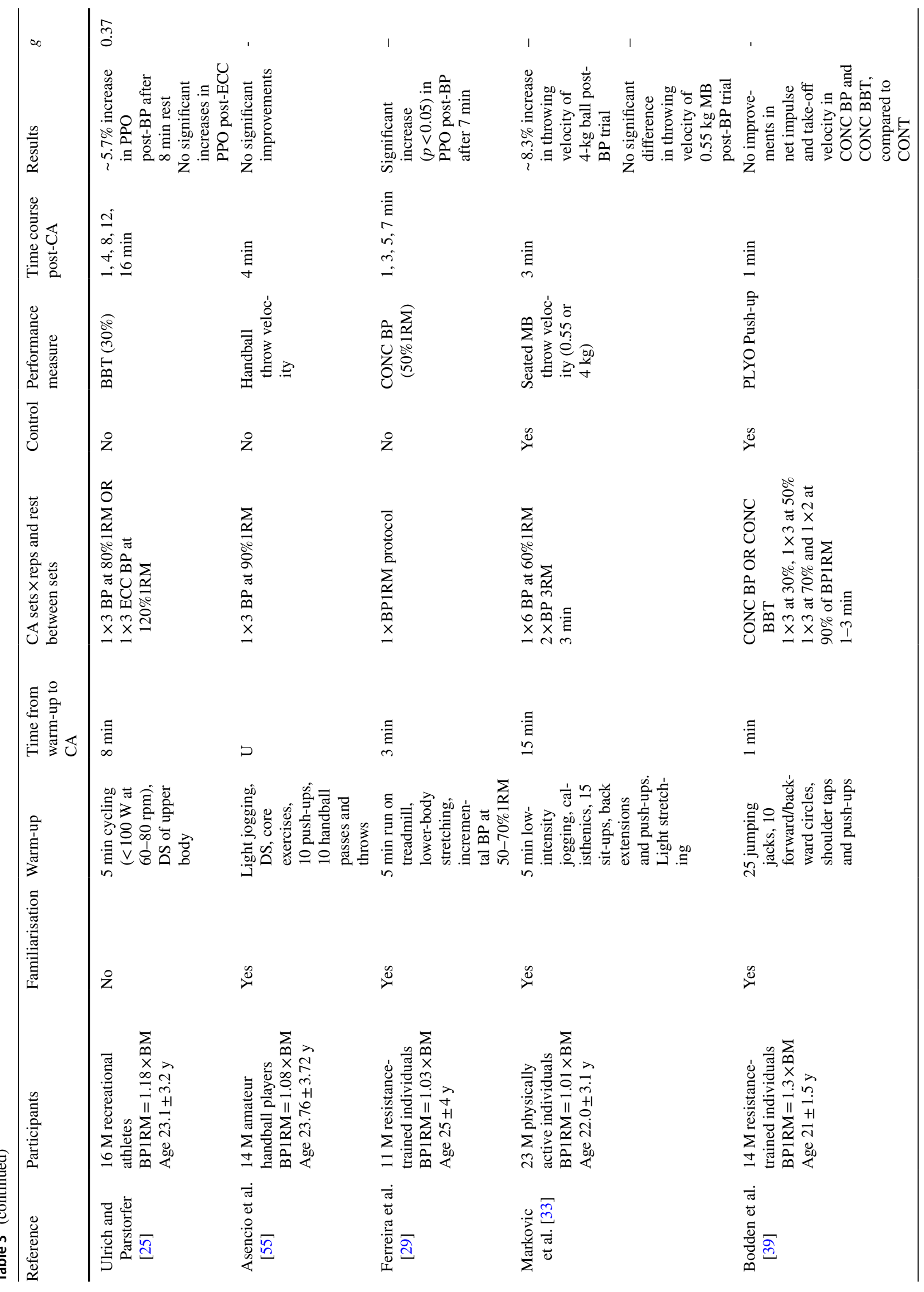




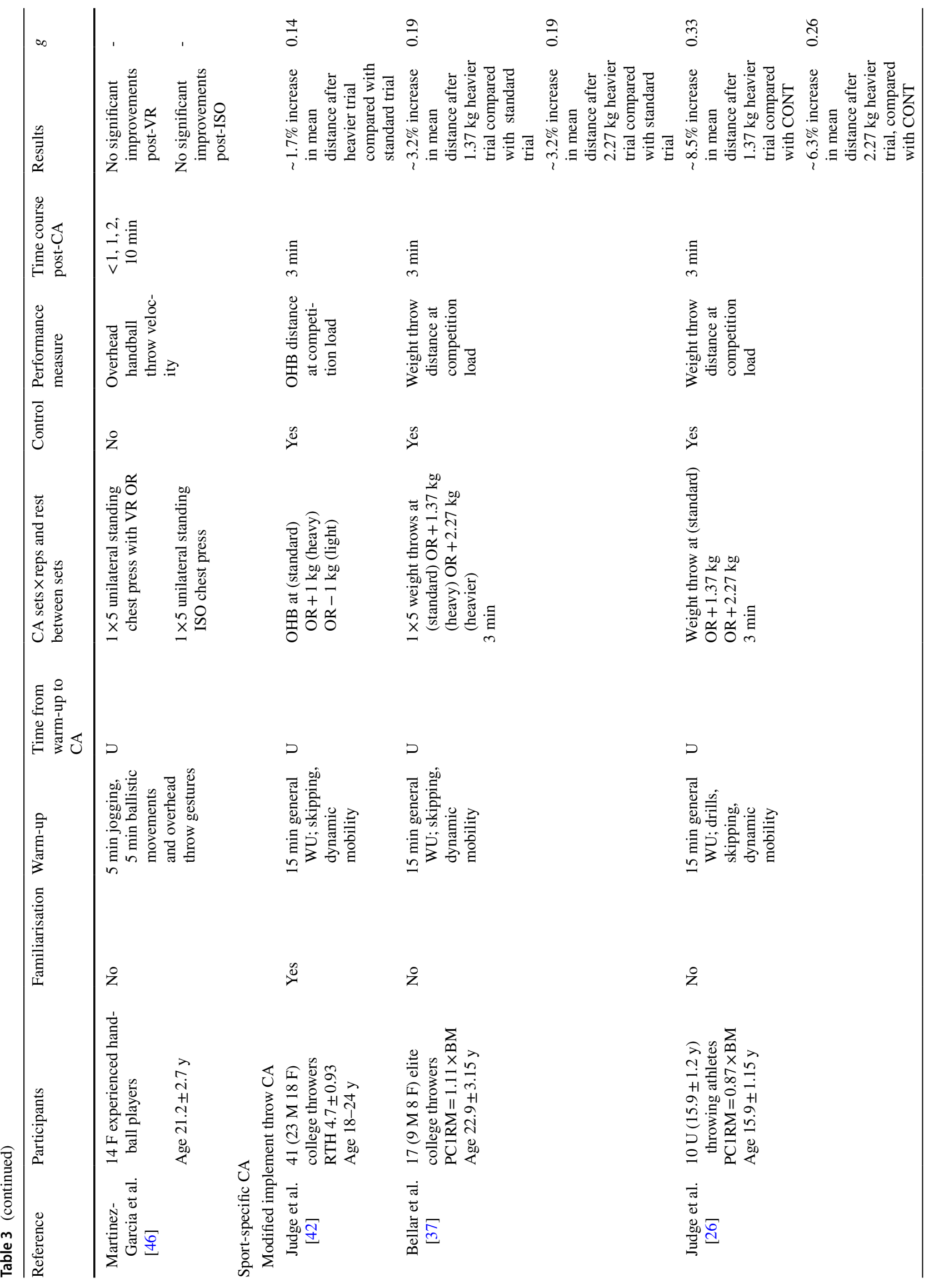




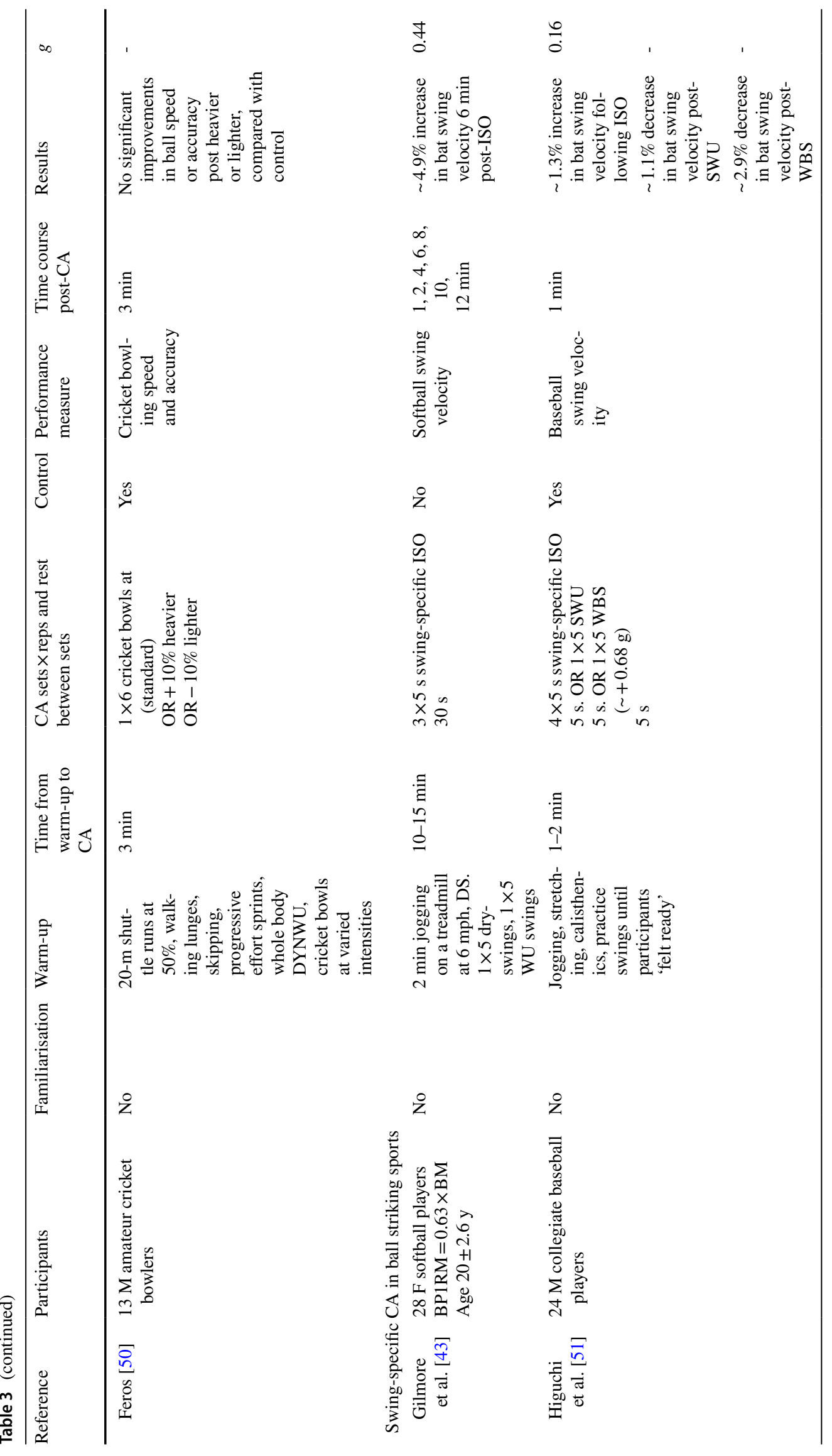




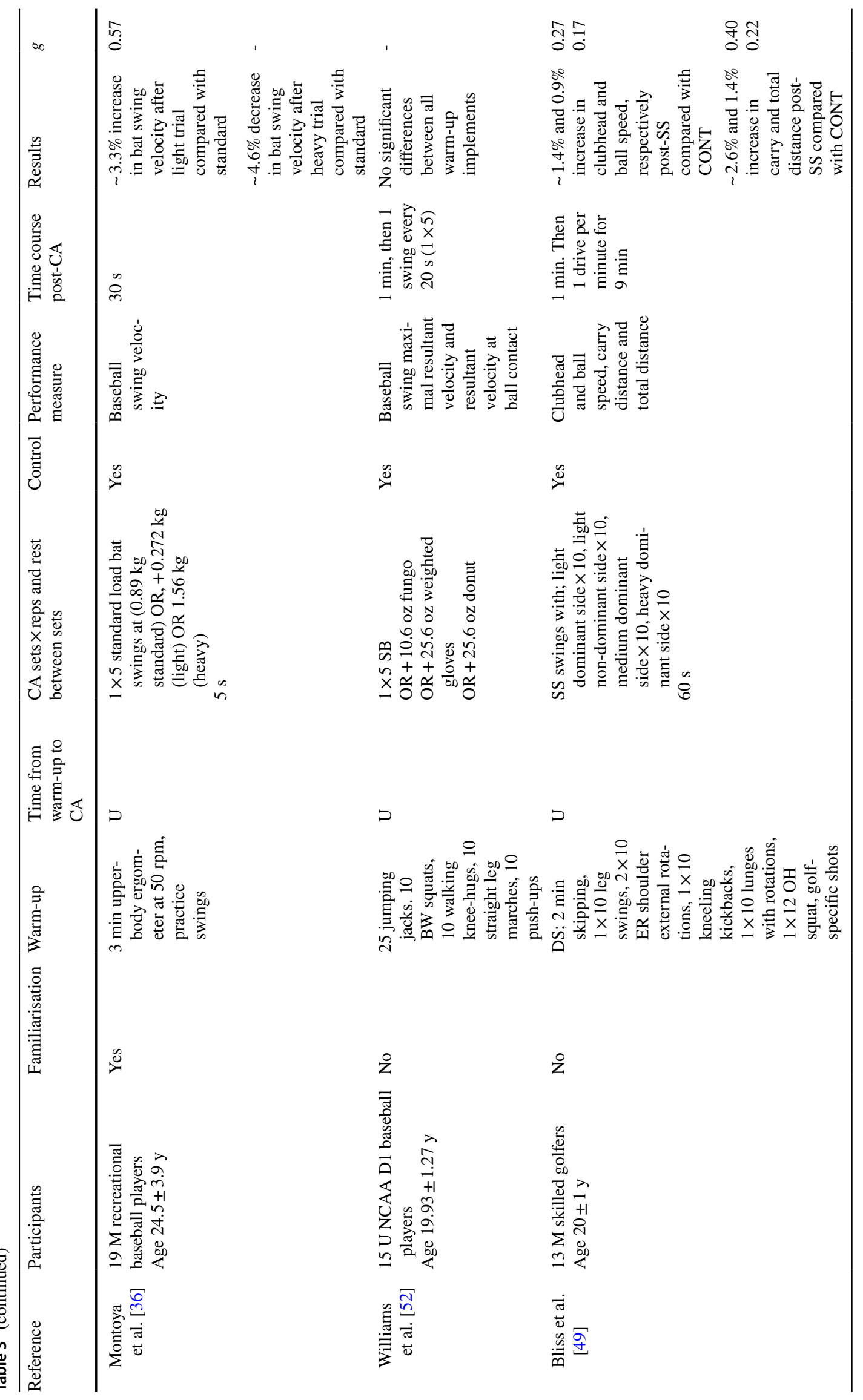




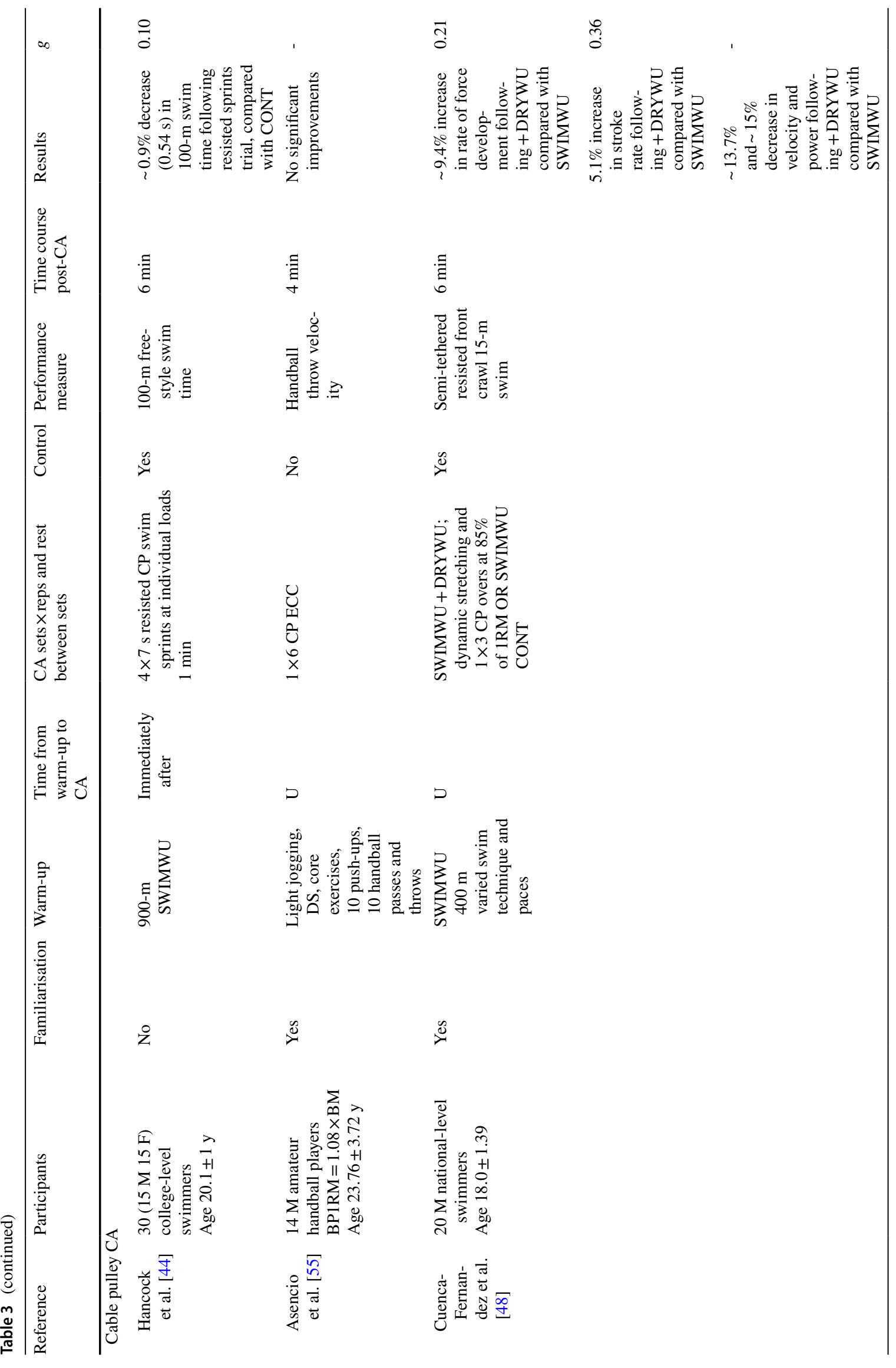




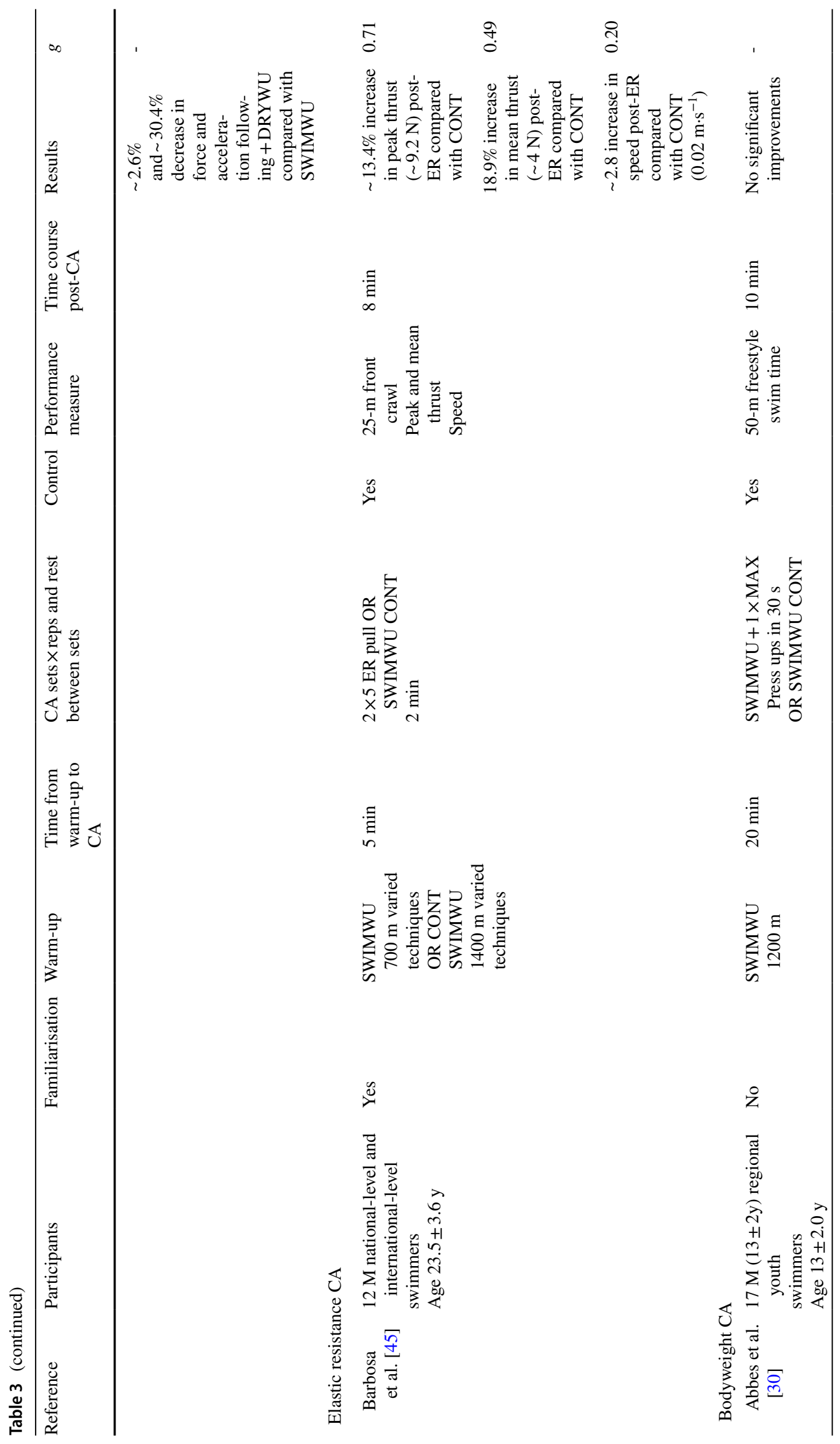




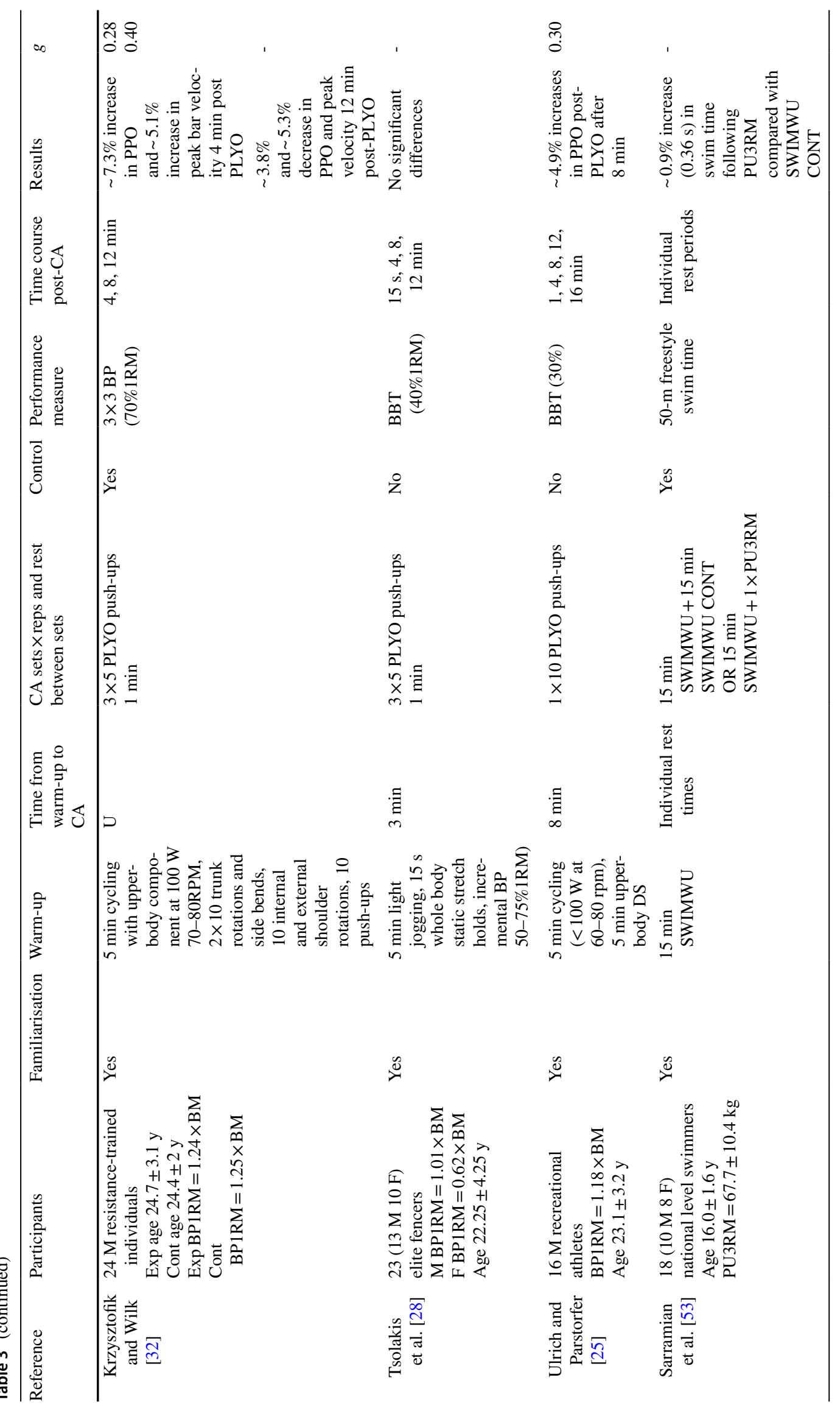




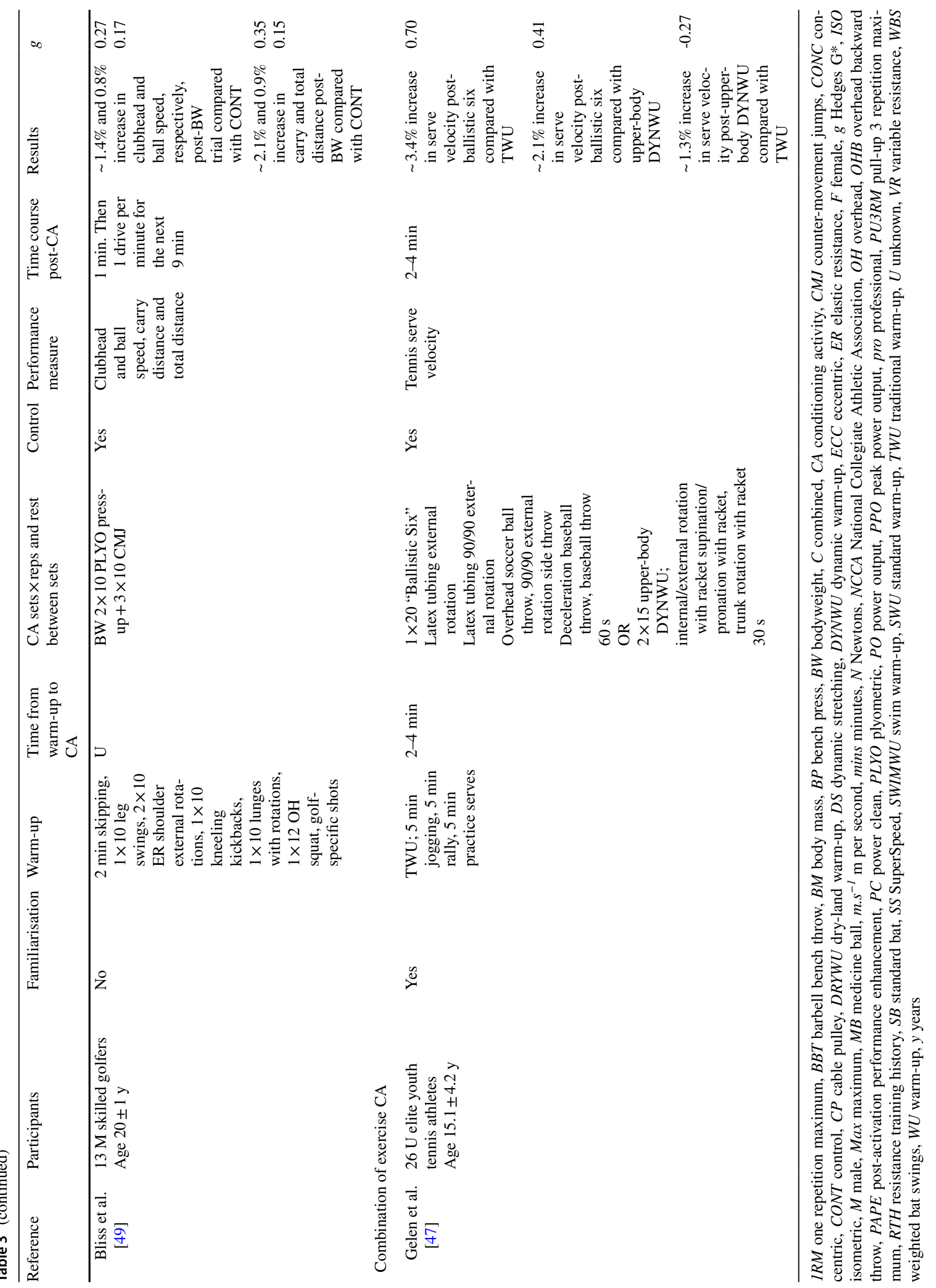




\subsection{Characteristics of Participants in the Included Studies}

Studies were published from 2005 to 2021 and included a total of 565 individual participants. This comprised 404 male individuals and 101 female individuals, the sex of 60 participants were not provided. The participants were largely of an athlete population (85\%), with an equal distribution of professional and amateur/recreational athletes as determined by the participant information disclosed by the study's authors. The other $15 \%$ were defined as resistance trained.

\subsection{Bench Press Variation Conditioning Activities}

Bench press variations were the most frequently utilised PAPE conditioning activity, with 14 studies including this type of activity. This comprised the bench press, eccentriconly bench press, concentric-only bench press and isometric bench press, in addition to the ballistic bench throw and variable resistance variations. All studies included low-volume protocols, from 1 to 3 sets, of 1-6 repetitions as seen in Table 3. Load varied across each bench press variation and is described in more detail in the following sections. Rest periods between consecutive conditioning sets, where applicable, also varied between studies. This typically ranged from $<1 \mathrm{~min}$ to $4 \mathrm{~min}$, whilst one study comprised individualised rest periods [27]. There was also disparity in the rest period from the cessation of the conditioning activity, and the following performance test between studies. Some authors monitored performance at single timepoints, whilst other authors reported data at multiple timepoints to identify the time course of PAPE. Overall, this ranged from $15 \mathrm{~s}$ to 24 min post-conditioning activity.

Small-to-moderate $(\mathrm{ES}=0.06-1.34)$ performance increases in ballistic bench throw power output following a bench press variation conditioning activity were reported in seven studies [25, 27, 35, 38, 40, 41, 54]. This translated to a peak improvement ranging from $\sim 3.3$ to $5.7 \%$. Conversely, two studies reported no significant improvements $[28,34]$ and one study reported a marginally detrimental effect [35]. An 4.3\% improvement in ballistic bench throw was reported $8 \mathrm{~min}$ following $3 \times 3$ bench press at $87 \% 1 \mathrm{RM}$ in professional rugby players [27]. Other studies found $8 \mathrm{~min}$ to be an optimal recovery duration to elicit PAPE following bench press at similar loads, where several timepoints were considered [25, 38, 41]. No improvements in the throwing velocity of amateur handball players were found following a bench press conditioning activity of 90\%1RM [55]. Similarly, no increases in throwing velocity of a light-weight $(0.55-\mathrm{kg})$ medicine ball were observed following several sets of bench press [33], though improvements $(\sim 8.9 \%)$ were observed in the $4-\mathrm{kg}$ medicine ball condition. No improvements were found in bench press power output when preceded by $3 \times 3$ bench press at $85 \% 1 \mathrm{RM}$ [31]. An increase in power output was observed in the concentric-only bench press, 7 min following a single set of bench press at $1 \mathrm{RM}$ [29]. Only a single study explored the use of a concentriconly bench press to improve subsequent ballistic bench throw in competitive rugby players [35]. The authors found that a single set of 3 repetitions at 3RM induced a small increase $(\sim 3.3 \%$; $\mathrm{ES}=0.15)$ in the ballistic bench throw power output. In contrast, concentric-only bench press did not improve subsequent upper-body plyometric performance [39]. No studies showed improvements in performance following an eccentric-only bench press conditioning activity $[25,35]$. No improvements in BBT power output were found following an isometric bench press conditioning activity of $3 \times 3 \mathrm{~s}$ [28]. In contrast, an $2.8 \%$; $\mathrm{ES}=0.14$ improvement in power output in the BBT was found following 1 repetition of isometric bench press of a 7-s duration [35]. A standing isometric chest press variation did not elicit PAPE in experienced handball players [46], observed as no improvements in overhead handball throwing velocity.

\subsection{Sport-Specific Conditioning Activities}

The following sub-sections highlight the results from studies where authors used sport-specific conditioning activities, comprising either the sporting action or a conditioning activity that shared biomechanical similarities to the performance test.

\subsubsection{Modified Implement Throw Conditioning Activities}

Three studies found that the use of overweight implement throws, 3 min prior to competition weight throws, elicits a performance enhancement, as observed by an increase in distance ( 1.7-8.5\%; ES =0.14-0.33) [26, 37, 42]. A single study found that a single set of six bowls of a heavier $(+10 \%)$ cricket ball did not improve accuracy or speed in subsequent cricket bowls, compared to a control trial [50]. Indeed, similar results were reported in the lighter ball $(-10 \%)$ condition [50].

\subsubsection{Swing-Specific Conditioning Activities in Ball Striking Sports}

Five studies were included in the review pertaining to ball striking sports [36, 43, 49, 51, 52]. Improvements in golf club and ball speed $(\sim 1.4 \% ; 0.9 \%)$ and carry and total distance $(\sim 2.6 \% ; 1.4 \%)$ were reported following a prior conditioning activity of light and heavy golf-specific implement swings [49]. Likewise, a single study reported an increase ( 3.3\%) in swing velocity after lighter baseball bat swings, compared with a standard bat [36]. On the contrary, three studies have shown a lack of improvement, and in some 
cases, a detrimental effect on performance, when prior swings are performed with a heavier bat [36, 51, 52]. Two studies $[43,51]$ have observed increases in bat swing velocity $(\sim 1.3-4.9 \%)$ following a conditioning activity of isometric bat swings.

\subsubsection{Cable Pulley Conditioning Activities}

Three studies explored the use of a cable pulley-type mechanism as a 'sport-specific' PAPE conditioning activity [44, $48,55]$. An improvement in swim time ( 0.9\%) was reported 6 min following a PAPE conditioning activity of $4 \times 7 \mathrm{~s}$ of resisted swim sprints [44]. One study found improvements in rate of force development ( 9.4\%) and stroke rate in a resisted front crawl swim, following a single set of three cable pullovers [48]. However, the same study reported decreases in several performance variables such as velocity, power, force and acceleration when compared with a control trial. No improvements in handball throwing velocity were reported following eccentric conical pulley activity in amateur handball players [55].

\subsubsection{Elastic Resistance Conditioning Activities}

Moderate improvements in peak $(\sim 13.4 \%$; $\mathrm{ES}=0.71)$ and mean $(18.9 \%$; ES $=0.49)$ thrust and speed $(\sim 2.8 \%$; ES 0.20$)$ were observed in international and national-level swimmers following a conditioning activity of elasticated pulls, compared with a control trial [45].

\subsection{Bodyweight Conditioning Activities}

Six studies included in the review investigated the efficacy of bodyweight PAPE conditioning activities $[25,28,30,32,49$, 53]. An $4.9 \%$ increase in power output in the ballistic bench throw was reported 8 min following a PAPE conditioning activity of $1 \times 10$ plyometric push-ups [25]. In contrast, a separate study found that $3 \times 5$ repetitions of the same conditioning activity failed to elicit performance improvements in the ballistic bench throw [28]. Another study reported improvements in power output and bar velocity in the bench press, following $3 \times 5$ plyometric push-ups [32]. A PAPE conditioning activity of $2 \times 10$ plyometric push-ups in addition to $3 \times 10$ counter-movement jumps elicited increases in clubhead $(\sim 1.4 \%$; ES $=0.27)$ and ball speed $(0.8 \%$; ES $0.17)$, and carry $(\sim 2.1 \%$; ES $=0.35)$ and total distance $(0.9 \%$; ES $=0.15)$ in skilled golfers, compared with a control trial [49]. The addition of a single set of 3RM pull-ups to a swimming warm-up did not induce performance benefits in 50-m freestyle swim time, compared to a control trial of the swimming warm-up only [53].

\subsection{Combination of Exercise Conditioning Activities}

A single study asked tennis players to perform a combination of exercises to explore whether such conditioning activity could induce performance improvements [47]. An upperbody dynamic warm-up elicited an $\sim 1.3 \%$ increase in tennis serve velocity when compared with a traditional warmup. Further improvements were found compared with the control trial, following a 'ballistic six' condition, whereby athletes performed several upper-body rotational ballistic movements.

\subsection{Study Results}

Table 3 displays the participant and experimental details, and the results of the literature pertaining to upper-body PAPE.

\subsection{Meta-analysis}

Because of the variations in the experimental design of the studies (randomised controlled trial and pre-post), a metaanalysis was appropriate for only one subset of studies that included a pre-post-test design; the bench press and ballistic bench throw (BBT) complex (Fig. 2). This was possible because of the similarity in conditioning protocols and outcome measure (power output in the ballistic bench throw).

Six studies investigated the effect of heavy $(\geq 80 \%)$ bench press on power output in a subsequent BBT $[25,27,35,38$, $40,41]$. The use of the heavy bench press as a conditioning activity had a positive effect $(p=0.03)$ on BBT performance compared with baseline ( $\mathrm{ES}=0.31$; CI 0.03-0.58) [Fig. 2] No heterogeneity was observed $\left(I^{2}=0 \%, p=0.98\right)$.

\section{Discussion}

This study aimed to perform a systematic review and metaanalysis on the efficacy of upper-body PAPE conditioning activities. Data suggest that several upper-body conditioning activities can be used to acutely improve upper-body performance. Findings varied between studies, at times because of differences in experimental designs and procedures, as discussed in later sections.

\subsection{Bench Press Variation Conditioning Activities}

As highlighted in the meta-analysis, the bench press with loads of $\geq 80 \% 1 \mathrm{RM}$ can be an effective conditioning activity to elicit improvements in subsequent power output in the BBT at 30-40\%1RM [25, 27, 35, 38, 40, 41]. Only one study [35] reported a marginally detrimental effect on BBT power output. Peak power output was typically observed between 
8 and 12 min post-conditioning activity; however, this varied because of the different timepoints considered. Where performance at multiple timepoints was considered, three studies reported a peak in BBT power output after 8 min rest [25, 38, 41], whilst one study reported a peak at $12 \mathrm{~min}$ [40]. All of the above studies implemented a bench press protocol of $\geq 80 \% 1 \mathrm{RM}$, which could suggest that a heavy load bench press conditioning activity requires a considerable recovery duration to induce PAPE. It should be noted, however, that loads were rarely directly compared in the literature. Liossis et al. [41] found 4 min was optimal recovery following bench press at $65 \% 1 \mathrm{RM}$, whilst 8 min was considered optimal for the $85 \% 1 \mathrm{RM}$ load. This again highlights the tradeoff between fatigue and PAPE. Similarly, 7 min following a bench press protocol at $90 \% 1 \mathrm{RM}$ was sufficient recovery to observe a significant increase in concentric-only bench press power output [29]. Coaches and practitioners may wish to use the bench press as a conditioning activity to evoke PAPE in subsequent BBT; however, as noted by Seitz and Haff [4], greater recovery durations may be needed where high loads are used.

The only study that utilised a heavy bench press protocol on subsequent bench press power output found no significant improvements [31]. However, the $3 \times 3$ protocol of $85 \% 1 \mathrm{RM}$ in that study was described as 'reps to failure', which is perhaps not synonymous with producing efforts of maximum power output. Further research is needed to analyse the effects of prior heavy bench press on subsequent bench press power output.

Bench press also failed to elicit improvements in handball throwing velocity [55]. Firstly, the biomechanical differences between the conditioning activity and the performance test are apparent [4], which may suggest the bench press may not transfer to acute task-specific performance improvements. A recent review found the greatest PAPE was typically observed $\geq 5$ min following high-to-moderate intensity conditioning activities [4]. It could, therefore, also be plausible that the single measurement at $4 \mathrm{~min}$ [55] may not have been sufficient recovery to reduce fatigue from the multiple repetitions of 90\%1RM bench press. The same limitations applied to a study by Martinez-Garcia et al. [46], who found no significant improvements in overhead handball throwing velocity following a standing chest press with variable resistance, with even shorter initial recovery durations. Markovic et al. [33] also found bench press produced non-significant differences in throwing performance 3 min following the conditioning activity, where the medicine ball load (0.55$\mathrm{kg}$ ) was similar to that of a handball. However, the latter study did find a significant improvement $(p=0.001 ; 8.3 \%)$ in throwing distance at the same rest period, with a heavier (4-kg) medicine ball. The bench press may have potential in improving sport-specific performance such as throwing, though this may be load specific [33]. The monitoring of only one timepoint in both studies could mask potential PAPE effects that manifest at greater recovery durations, which may mislead the coach or practitioner on the efficacy of such conditioning activities. However, it is worth noting that several testing intervals may also negatively affect subsequent performance due to fatigue, therefore requiring careful consideration.

Only a single study [35] explored the use of concentriconly bench press as a conditioning activity to improve BBT power output, evidencing potential effectiveness. Similarly, only one study explored the use of concentric-only bench press on subsequent plyometric activity [39], finding it may not be an effective method to acutely improve performance in the plyometric push-up. Post-activation performance enhancement was monitored at only one timepoint following the conditioning activities in the two studies [35, 39], $12 \mathrm{~min}$ and $1 \mathrm{~min}$, respectively. Again, it is conceivable that performance improvement could manifest at later timepoints, when considering the time course of PAPE is thought to last for several minutes [4]. In the initial minutes following the conditioning activity, other mechanisms such as PAP may be the basis of performance improvement [5], suggesting the $1 \mathrm{~min}$ recovery period in the study by Bodden and colleagues is inappropriate in relation to PAPE. This is especially true when reflecting on the incremental high-volume protocol involved [39]. The lack of studies exploring the efficacy of concentric-only bench press to induce PAPE suggests the evidence is unclear.

The evidence presented in this review suggests that eccentric-only bench press does not induce a PAPE effect in BBT power output $[25,35]$. Both studies reported no significant differences as a result of the conditioning activity. Though high loads have been demonstrated to induce PAPE throughout this review, the supramaximal nature $(120 \% 1 \mathrm{RM})$ of the conditioning activity in the study by Ulrich and Parstorfer [25] may have induced too much fatigue, thus suppressing PAPE. This is despite the lower number of overall reps $(1 \times 3)$ performed compared with bench press PAPE studies. Esformes et al. [35] used a lighter load, equivalent to $3 \mathrm{RM}$, and found only a very marginal increase $(0.8 \%)$ in power output. Therefore, the PAPE-inducing capability of the eccentric-only bench press on the BBT is not currently supported by the literature.

The two studies that included an isometric variation presented conflicted findings [28, 35]. Tsolakis et al. [28] found no significant differences in BBT power output following isometric bench press. Further analysis revealed that the female athletes possessed relative strength levels (bench press $1 \mathrm{RM}$ as a percentage of body mass) of 0.62 , noticeably lower than the 1.01 achieved by the male athletes in the same study. As strength level is a modulating factor of the PAPE response [4], the large differences in strength levels could explain the apparent lack of performance improvement. 


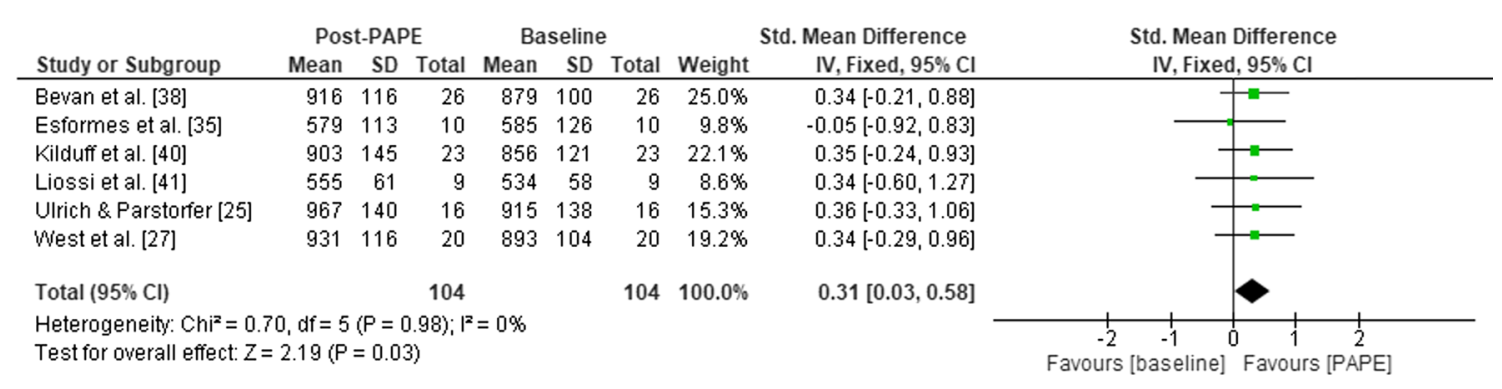

Fig. 2 Forest plot for power output in the barbell bench throw pre and post a bench press conditioning activity at $\geq 80 \% 1 \mathrm{RM}$. $C I$ confidence interval, $I V$ inverse variance, $P A P E$ post-activation performance enhancement, $S D$ standard deviation, Std standard

Specifically, stronger athletes are able to achieve greater levels of PAPE $(\mathrm{ES}=0.41)$, compared with their weaker counterparts $(\mathrm{ES}=0.32)$ [4]. It has been suggested that an increased level of strength may make an individual more resistant to fatigue following a conditioning activity, thus responding more favourably than weaker athletes [4].

In contrast, Esformes et al. [35] did identify significant improvements in subsequent BBT power output, where participants possessed overall relative strength levels (bench press 1RM as a percentage of body mass) of 1.1 in the bench press. Additionally, the latter study included only one maximal voluntary contraction, whereas Tsolakis et al. [28] included three. Differing protocols and the ensuing fatigue may contribute to the varied results, though the overall contraction time was similar in both studies. The lack of research makes it difficult to infer the effectiveness of an isometric bench press conditioning activity in inducing an acute performance benefit in the BBT. Efficacy may be contingent on athlete strength levels, and by association, sex.

West et al. [27] reported increases in BBT power output, 8 min following a $3 \times 3$ BBT conditioning activity at $30 \% 1 \mathrm{RM}$. In contrast, separate trials of $1 \times 5 \mathrm{BBT}$ at various loads $\geq 50 \% 1 \mathrm{RM}$ did not influence BBT power output after 4 min rest [34]. The disparity between the two studies may be explained by the increased recovery time in the former study. A single set of 3 repetitions of BBT at $30 \%$ did not elicit any differences in subsequent BBT performance [54]. Interestingly, in the same study, a contrast protocol of $1 \times 3 \mathrm{BBT}$ at $30 \% 1 \mathrm{RM}$, followed by $1 \times 5$ at $60 \% 1 \mathrm{RM}$ load did produce an improvement in power output at $3 \mathrm{~min}$ $(8.7 \%)$ and $5 \mathrm{~min}(10.4 \%)$, respectively. The differences at the two timepoints again support the notion of optimal postintervention timing. The evidence suggests that the BBT as part of a resistance exercise contrast or complex set may be a promising conditioning method to induce PAPE, and thus, improve subsequent performance in the same activity. However, as is a common theme throughout, more research is required to confirm this.

Further research could explore whether bench press variations can acutely influence task-specific or sporting performance. This would enable practitioners and coaches to determine whether it has appropriate inclusion as a precompetition warm-up conditioning activity for athletes. Though, as previously discussed, biomechanical specificity and logistical issues mean more sport-specific PAPE conditioning activities could be better alternatives for athletes.

\subsection{Sport-Specific Conditioning Activities}

Select studies explored the use of movement-specific combinations that involve the sporting action as the conditioning activity and performance test, thus greater specificity to sporting performance. These complexes can be used more widely for athletes outside of a weight room or laboratory, for example, within the constraints of the competition warmup environment and are described in the sections below.

\subsubsection{Modified Implement Throw Conditioning Activities}

The current data suggest that warming up with overweight implements could potentially improve subsequent throwing for distance performance; however, the previous literature suggests there may be a limit to performance benefits at increased loads [26, 37, 42]. A plausible reason for the improvement could be due to the conditioning activities and the performance tests being either identical or at least sharing biomechanical similarities [4]. It is worth noting that although the participants were trained in their respective throwing disciplines, strength levels in the studies varied $[26,37]$, whilst one study did not report this information [42]. All studies shared a 3 min recovery period between the cessation of the conditioning activity, and the performance measure. Whilst this suggests that a 3 min period may be sufficient to elicit a performance improvement following a throwing conditioning activity, the time course of PAPE is purported to last several minutes [4]. Thus, the lack of monitoring at several timepoints may underestimate a 'peak' in the PAPE effect. Though, it is again worth noting the potential negative performance effects that could arise from excessive testing intervals. Nevertheless, on current 
evidence, warm-up throws with a marginally overweight implement compared with the competition standard seem to be an effective method of improving competition throwing performance where increased distance is the primary goal. Therefore, throwing coaches could consider adopting this well-practiced strategy either as a pre-competition conditioning activity or in-between throws in training, once individual athlete responsiveness has been established. In contrast, the use of a heavy cricket ball, and indeed a lighter cricket ball, did not yield any performance improvement in cricket bowling speed and accuracy [50]. Again, the latter study included a short recovery duration of $3 \mathrm{~min}$ between the conditioning activity and the subsequent performance test. Likewise, participant strength levels were not reported.

\subsubsection{Swing-Specific Conditioning Activities in Ball Striking Sports}

Five studies were included in the review pertaining to ball striking sports [36,43,49,51,52]. Bliss et al. [49] reported improvements in speed and distance in the golf swing, $1 \mathrm{~min}$ following prior swings with light and heavy implements, compared with a control trial. The study by Bliss et al. [49], and indeed all of the swing-specific sports included in the review, comprised PAPE conditioning activities that shared clear biomechanical specificity to the performance test in terms of the movement pattern. Bat swing-specific isometric contractions of a 5-s duration seemingly induce a PAPE effect on subsequent swing velocity [43, 51]. Gilmore et al. [43] demonstrated a PAPE effect following $3 \times 5$ bat swingspecific isometric contractions, in a group of athletes that are not considered to be the strongest, whereas Higuchi et al. [51] did not report strength characteristics. Interestingly, the latter study showed an $1.3 \%$ increase in swing velocity after just $1 \mathrm{~min}$ of rest post-conditioning activity. The evidence on the efficacy of weighted swings would suggest it does not improve, and in some cases, may even be detrimental to subsequent swing velocity $[36,51,52]$. Where Bliss et al. [49] reported improvements post 'heavy' swings, this was actually of a similar mass to that of a standard golf club, and was part of a protocol of contrasting implements. Montoya et al. [36] reported an increase (3.3\%) in swing velocity after lighter bat swings, compared with a standard bat. All studies pertaining to swing-specific conditioning activities in ball striking sports had administered the initial post-performance test within $1 \mathrm{~min}$ of the conditioning activity. Considering the proposed time course of PAPE and the possible coexistence of PAP and PAPE $[5,16]$, any performance improvements may not necessarily be solely attributed to PAPE. Despite the small body of work in the area, the use of bat swing-specific conditioning activities in the form of isometric contractions appear to be a useful method for improving subsequent swing velocity. This method has been applied in baseball and softball, though it may offer a novel method for the coach and practitioner across other sports that require upper body distal point velocity, such as throwing or punching. The use of lighter weighted swings or contrasting light-heavy swings may be useful in improving subsequent swing-specific performance [36, 49]; however, more research should explore the efficacy of this method.

\subsubsection{Cable Pulley Conditioning Activities}

Three studies explored the use of a cable pulley-type mechanism as a 'sport-specific' PAPE conditioning activity. Asencio et al. [55] did not find any improvements in handball throwing velocity after eccentric conical pulley activity, with the authors stating this was possibly due to the short recovery time of 4 min. Hancock et al. [44] implemented a longer recovery duration of $6 \mathrm{~min}$ following the swimming-specific mode and found an $\sim 0.8 \%$ reduction in swim sprint time, corresponding to just under a 1-s improvement. CuencaFernandez et al. [48] also administered a 6 min recovery period to national-level swimmers post-dynamic stretching and $1 \times 3$ cable pull overs at $85 \%$ of $1 \mathrm{RM}$. Compared with a control trial of a swimming-only warm-up, rate of force development and stroke rate improved in the 15-m front crawl. Interestingly, the conditioning activity produced a negative effect on variables such as velocity and distance covered. More research is needed to understand the potential benefits of the cable pulley or similar resistance, as a PAPEinducing conditioning activity.

\subsubsection{Elastic Resistance Conditioning Activities}

Only one study was included in the review that explored the efficacy of a PAPE conditioning activity with elastic resistance [45]. Increases in the thrust and speed of international and national-level swimmers' 25 -m front crawl performance was reported following $2 \times 5$ elasticated pulls performed 8 min prior. The inclusion of only one study does perhaps show an under-utilisation of elastic resistance to induce PAPE. Elastic resistance has successfully induced a PAPE effect in combat-specific actions [56, 57]; however, because of the lower-body elements of the conditioning activities, the relevant studies were not included in this review. Therefore, future research should further explore the efficacy of upper-body elastic resistance activity in inducing PAPE in a variety of sports.

\subsection{Bodyweight Conditioning Activities}

Sarramian et al. [53] compared the effects of a swimming warm-up inclusive of $1 \times 3$ pull-ups at $3 \mathrm{RM}$, to a control trial of just a swimming warm-up. The findings showed the addition of the pull-up conditioning activity did not improve 
50-m freestyle swim performance to a greater extent than the swimming warm-up, despite the inclusion of individual rest times. The only study to explore the PAPE-inducing effect of the push-up, included a $30-\mathrm{m}$ freestyle swim as the performance test [30]. The authors found no significant improvements compared to a control trial of rest. A number of factors could explain the lack of performance improvement. Firstly, the protocol was reported as a 30-s maximal effort. This conditioning activity may induce levels of fatigue that would suppress any possible PAPE effect. Indeed, the authors reported mean pre-swim blood lactate levels of $3.6 \pm 0.9 \mathrm{mmol} . \mathrm{l}^{-1}$ during the push-up trial, compared with $1.9 \pm 8 \mathrm{mmol}^{-1}$ at the same point in the control. There is also arguably little biomechanical specificity between the push-up and freestyle swim technique; however, it should be noted that it is not a requirement for the conditioning activity to directly mimic the sporting activity [11]. Lastly, no information on the strength levels of participants was provided by the authors.

Plyometric versions of the push-up have received more focus in the literature. Ulrich and Parstorfer [25] found the plyometric push-up to be an effective conditioning activity to improve subsequent BBT power output by $\sim 4.9 \%$ after $8 \mathrm{~min}$. Both exercises share similar stretch-shortening cycle principles, whereby a rapid eccentric loading is followed by a rapid concentric push [58]. This again perhaps highlights the importance of biomechanical specificity. Other authors [28] reported only a marginal and non-significant increase in BBT performance, when preceded by a plyometric push-up protocol, comprising the same recovery time implemented by Ulrich and Parstorfer [25]. However, the former study included both female and male participants, with female participants exhibiting considerably lower strength levels, resulting in markedly greater strength levels in the study by Ulrich and Parstorfer [25]. Krzystofik and Wilk [32] found increases in peak power output and bar velocity 4 min post $3 \times 5$ plyometric push-ups compared with a control trial; however, both variables were weaker than the control trial at 12 min post. Krzystofik and Wilk [32] also reported considerably greater strength levels in their study, compared with that of Tsolakis et al. [28]. As PAPE seemingly manifests in a greater magnitude in stronger athletes [4], this could partly explain the different magnitudes of PAPE observed between the studies. Additionally, Tsolakis et al. [28] instructed participants to complete 15 overall repetitions compared with ten in the study by Ulrich and Parstorfer [25]. Plyometric push-ups performed alongside counter-movement jumps elicited small increases in golf drive speed and distance compared with a control trial [49]. The inclusion of the lower body element, however, makes it difficult to attribute the performance increase to the plyometric push-up.

In summary, it is not yet possible to infer the usefulness of push-ups as a conditioning activity to induce PAPE.
However, the findings of three studies [25, 28, 32] suggest that the plyometric variation of the push-up may have potential as a conditioning activity to induce PAPE in the bench press, BBT or golf drive performance.

\subsection{Combination of Exercise Conditioning Activities}

One study included in the review was categorised on its own, due to the combination of many types of activity [47]. Gelen et al. [47] found increases in tennis serve velocity following an upper-body dynamic warm-up, inclusive of movements with the tennis racket. Interestingly, even greater benefits were found following a 'ballistic 6' protocol, whereby the tennis athletes performed a combination of upper-body rotational movements with elastic resistance and implement throws. This suggests a conditioning activity inclusive of ballistic movement may induce PAPE, as seen in the previously discussed sport-specific sections. It is, however, difficult to infer which activities are responsible for the performance improvements.

\section{Limitations}

This review has demonstrated the potential of many upperbody exercises in inducing PAPE, thus improving subsequent athletic performance. The following sections briefly summarise the limitations of the included studies and that of the current review, with accompanying recommendations on potential future research direction and design.

\subsection{Limitations of the Included Studies}

Whilst PAPE and fatigue can coexist [59], the magnitude of the two can be dependent on many factors, including but not limited to, the type of conditioning activity and the recovery duration. Unfortunately, the body of literature on upper-body PAPE has typically consisted of varied conditioning activity and performance test complexes, in addition to a range of recovery durations, making it difficult to directly compare findings. Researchers should ensure an appropriate time course is applied in monitoring the response to a conditioning activity, particularly considering PAPE could last for several minutes $[4,5]$. This would also allow for the analysis of individual PAPE responses [13], whereby an optimal recovery duration could be implemented for individual athletes. The reporting of group and individual characteristics such as age, strength level and training history, to name a few, would allow for a greater analysis of the relationship between these factors, and the PAPE response in responders and non-responders.

An element that has often been ignored in the pre-post study designs is the effect of the warm-up on the apparent 
PAPE effect. The many benefits of a warm-up to athletic performance has been established previously [1-3]. If it is currently accepted that the mechanisms of warm-up and PAPE are similar [5], then it is difficult to isolate the two elements and correctly attribute a performance enhancement in prepost study designs, in the absence of a control trial. There are several examples in the literature where details of the warm-up or the recovery durations between the warm-up, conditioning activity and baseline tests were scarce. Future studies should include a pure control trial inclusive of a standardised warm-up and an experimental trial inclusive of a standardised warm-up and conditioning activity, so that potential performance improvements that may be observed can be attributed to the conditioning activity, and not confounding variables [5]. An additional important consideration for the warm-up that was neglected in many studies was a lack of sport or 'task-specific' activity, which Blazevich and Babault [5] note may invalidate the real-world applicability of the warm-up.

The current review revealed 11 studies did not include details on familiarisation trials, whereby participants initially become accustomed to the conditioning activities and performance tests to be included in the experimental trials to minimise potential learning effects [60, 61]. Lastly, whilst research exploring the effectiveness of upper-body conditioning activities on isolated sporting or exercise tasks exists, there is seemingly a lack of research exploring the PAPE effects on continuous competitive or simulated match play. For a deeper understanding of how PAPE can impact athletic performance, future research could consider monitoring the effects of PAPE on performance to competitive and simulated situations.

\subsection{Limitations of the Current Review}

The majority of studies in the bench press and BBT complex meta-analysis comprised pre-post observational designs; therefore, the pre-post standardised mean difference of studies could be calculated for the meta-analysis. The issues of pre-post-test standardised mean difference to indicate treatment effects in meta-analyses have been highlighted in recent research, primarily because of an increased risk of biased outcomes [62]. This can result in less reliable information being calculated about the effects of an intervention, as it only calculates the change within one group. Specifically, pre-post-test designs are not independent of each other, and can be influenced by factors external to the intervention [62]. Notwithstanding the above issues, the authors appreciate that this type of analysis may still be considered useful to the coach and practitioner in the absence of randomised controlled trials, providing the data are interpreted with caution. Additionally, because of the large variations in the methodological approach between studies, meta-analyses of the other conditioning activity classifications were not possible. Future research must aim for greater uniformity in its methodologies. This would assist in the easier interpretation of findings for the coach, practitioner and the meta-analyst.

\section{Conclusions and Practical Applications}

The current review highlights several upper-body conditioning activities that can potentially induce a PAPE effect on athletic performance. Bench press was the most frequently used conditioning activity, proving an effective method to induce a $3.9-5.7 \%$ increase in BBT power output following 8-12 min recovery, where bench press loads were between 80 and $87 \% 1 \mathrm{RM}$. This may offer the coach and practitioner a useful complex to improve athlete upper-body strength and power. The eccentric bench press, however, seemingly does not improve subsequent BBT performance, and may indeed impair performance when prescribed at supramaximal loads. Further research is needed to identify the efficacy of other bench press variations such as concentric only, isometric, and ballistic on subsequent ballistic, plyometric and throwing performance, as the literature is currently conflicted. This is important for the coach and practitioner, as understanding whether bench press variations transfer to improved 'sport-specific' movements may influence potential warm-up or resistance training complex prescription, in non-powerlifting athletes.

This review supports the prescription of overweight implement throws, prior to competition throw performance where the aim is for maximum distance. Coaches and practitioners could utilise this method in the pre-competition warm-up with throwing athletes, though they should consider whether there will inherently be a weight limit, whereby an increase in the overweight throw implement past a specific threshold may yield diminishing returns. Likewise, this review found movement-specific bat swing activity, in the form of lighter-weighted bat swings and isometric swings, could be promising strategies to acutely improve swing velocity. This strategy could perhaps improve distal point velocity, which could extend the application to striking sports such as boxing, whereby peak fist velocities is an important factor in effective punching.

Other movement-specific complexes that could have potential include resisted activity with a cable pulley type mechanism, select bodyweight activity and elastic resistance, though there is currently either conflicting results or a lack of literature on these conditioning activities. When volume is kept relatively low, this review also highlights the potential efficacy of the plyometric push-up, in improving subsequent ballistic performance in well-trained athletes.

Movement-specific combinations appear to be more successful in producing a performance improvement, in 
agreement with recent reviews on whole-body acute PAPE $[4,16]$. The coach and practitioner may wish to utilise some of the upper-body conditioning activities highlighted in this current review. Coaches and practitioners should, however, interpret the data with caution, owing to the many limitations in the previous literature that are highlighted throughout. To combat this, the authors have provided a very brief guidance on how future PAPE research could be improved. It is hoped this may assist in a greater uniformity in the methodological approach, a wider appreciation of potentially effective exercise modes outside of powerlifting techniques, and thus, a greater understanding of PAPE efficacy for coaches and practitioners.

\section{Declarations}

Funding No funding was received for this project.

Conflict of interest Mitchell Finlay, Craig Bridge, Matt Greig and Richard Page declare that they have no conflicts of interests relevant to the content of this review.

Ethics approval Not applicable.

Consent to participate Not applicable.

Consent for publication Not applicable.

Availability of data and material The datasets analysed during the current study are available from the corresponding author on reasonable request.

\section{Code availability Not applicable.}

Author contributions MF and RP contributed to the study concept and design. MF, RP and CB performed the literature search. MF and RP performed the data extraction and quality assessment. MF wrote the first draft of the manuscript. RP, CB and MG critically revised the draft and final versions of the manuscript. All authors approved the final manuscript.

Open Access This article is licensed under a Creative Commons Attribution 4.0 International License, which permits use, sharing, adaptation, distribution and reproduction in any medium or format, as long as you give appropriate credit to the original author(s) and the source, provide a link to the Creative Commons licence, and indicate if changes were made. The images or other third party material in this article are included in the article's Creative Commons licence, unless indicated otherwise in a credit line to the material. If material is not included in the article's Creative Commons licence and your intended use is not permitted by statutory regulation or exceeds the permitted use, you will need to obtain permission directly from the copyright holder. To view a copy of this licence, visit http://creativecommons.org/licenses/by/4.0/.

\section{References}

1. Fradkin A, Zazryn T, Smoliga J. Effects of warming-up on physical performance: a systematic review with meta-analysis. J Strength Cond Res. 2010;24(1):140-8.
2. Jeffreys I. Warm-up revisited: the ramp method of optimizing warm-ups. Prof Strength Condition. 2007;6:12-8.

3. Bishop D. Warm up I. Sports Med. 2003;33(6):483-98.

4. Seitz LB, Haff G. Factors modulating post-activation potentiation of jump, sprint, throw, and upper-body ballistic performances: a systematic review with meta-analysis. Sports Med. 2016;46(2):231-40.

5. Blazevich AJ, Babault N. Post-activation potentiation versus post-activation performance enhancement in humans: historical perspective, underlying mechanisms, and current issues. Front Physiol. 2019. https://doi.org/10.3389/fphys.2019.01359.

6. Cormier P, Freitas TT, Rubio-arias JA, Alcaraz PE. Complex and contrast training: does strength and power training sequence affect performance-based adaptations in team sports? A systematic review and meta-analysis. J Strength Cond Res. 2020;34(5):1461-79.

7. Tillin NA, Bishop D. Factors modulating post-activation potentiation and its effect on performance of subsequent explosive activities. Sports Med. 2009;39(2):147-66.

8. Cuenca-Fernandez F, Smith IC, Jordan MJ, Macintosh BR, LopezContreras G, Arellano R, et al. Nonlocalized postactivation performance enhancement (PAPE) effects in trained athletes: a pilot study. Appl Physiol Nutr Metab. 2017;42(10):1122-5.

9. Prieske O, Behrens M, Chaabene H, Granacher U, Maffiuletti N. Time to differentiate postactivation "potentiation" from "performance enhancement" in the strength and conditioning community. Sports Med. 2020;50(9):1559-65.

10. Boullosa D. Post-activation performance enhancement strategies in sport: a brief review for practitioners. Hum Mov. 2021;22:101-9.

11. Boullosa D, Beato M, Lacono AD, Cuenca-Fernandez F, Doma K, Schumann M, et al. A new taxonomy for postactiviation potentiation in sport. Int J Sports Physiol Perform. 2020. https://doi.org/ 10.1123/ijspp.2020-0350.

12. Smith I, Macintosh BR. A comment on "A new taxonomy for postactivation potentiation in sport." Int J Sports Physiol Perform. 2020. https://doi.org/10.1123/ijspp.2020-0586.

13. Healy R, Comyns T. Application of postactivation potentiation methods to improve sprint speed. Strength Cond J. 2017;39(1):1-9.

14. Choon NG, Chen SE, Lum D. Inducing postactivation potentiation with different modes of exercise. Strength Cond J. 2020;42(2):63-81.

15. Wilson JM, Duncan NM, Marin PJ, Brown LE, Loenneke JP, Wilson SMC, et al. Meta-analysis of postactivation potentiation and power: effects of conditioning activity, volume, gender, rest periods, and training status. J Strength Cond Res. 2013;27(3):854-9.

16. Izquierdo $\mathrm{M}$, Hakkinen $\mathrm{K}$, Gonzalez-Badillo JJ, Ibanez J, Gorostiaga EM. Effects of long-term training specificity on maximal strength and power of the upper and lower extremities in athletes from different sports. Eur J Appl Physiol. 2002;87(3):264-71.

17. Moher D, Liberati A, Tetzlaff J, Altman DG. Preferred reporting items for systematic reviews and meta-analyses: the PRISMA statement. PLoS Med. 2009. https://doi.org/10.1371/journal. pmed.1000097.

18. PEDro. PEDro scale, 1999. https://pedro.org.au/wp-content/uploa ds/PEDro_scale.pdf. Accessed 17 Oct 2019.

19. Krzysztofik M, Wilk M, Stastny P, Golas A. Post-activation performance enhancement in the bench press throw: a systematic review and meta-analysis. Front Physiol. 2021;15(11):598628. https://doi.org/10.3389/fphys.2020.598628.

20. Hedges LV, Olkin I. Statistical methods for meta-analysis. St. Louis (MO): Elsevier Science; 2014. http://qut.eblib.com.au/ patron/FullRecord.aspx?p=1901162. Accessed 30 Sept 2021. 
21. Morris SB. Estimating effect sizes from pretest-posttest-control group designs. Organ Res Methods. 2008;11:364-86. https://doi. org/10.1177/1094428106291059.

22. Cohen J. Statistical power analysis for the behavioral sciences. 2nd ed. Routledge; 1988.

23. Review Manager (RevMan) [Mac]. Version 5.4. The Cochrane Collaboration; 2020.

24. Deeks JJ, Higgins JPT, Altman DG, editors. Chapter 10. Analysing data and undertaking meta-analyses. In: Higgins JPT, Thomas J, Chandler J, Cumpston M, Li T, Page MJ, et al., editors. Cochrane handbook for systematic reviews of interventions, version 6.2 (updated February 2021). Cochrane, 2021. www.train ing.cochrane.org/handbook. Accessed 15 Nov 2021.

25. Ulrich G, Parstorfer M. Effects of plyometric versus concentric and eccentric conditioning contractions on upper-body postactivation potentiation. Int J Sports Physiol Perform. 2017;12(6):736-41.

26. Judge LW, Bellar D, Judge M. Efficacy of potentiation of performance through overweight implement throws on male and female high-school weight throwers. J Strength Cond Res. 2010;24(7):1804-9.

27. West DJ, Cunningham DJ, Crewther BT, Cook CJ, Kilduff LP. Influence of ballistic bench press on upper body power output in professional rugby players. J Strength Cond Res. 2013;27(8):2282-7.

28. Tsolakis C, Bogdanis GC, Nikkolaou A, Zacharogiannis M. Influence of type of muscle contraction and gender on postactivation potentiation of upper and lower limb explosive performance in elite fencers. J Sports Sci Med. 2011;10(3):577-83.

29. Ferreira SLDA, Panissa VLG, Miarka B, Franchini E. Postactivation potentiation: effect of various recovery intervals on bench press power performance. J Strength Cond Res. 2012;26(3):739-94.

30. Abbes Z, Chamari K, Mujika I, Tabben M, Bibi KW, Hussein $\mathrm{AM}$, et al. Do thirty-second post-activation potentiation exercises improve the 50-m freestyle sprint performance in adolescent swimmers? Front Physiol. 2018;9:1464. https://doi.org/10.3389/ fphys.2018.01464.

31. Krzysztofik M, Wilk M, Filip A, Zmijewski P, Zajac A, Tufan JJ. Can post-activation performance enhancement (PAPE) improve resistance training volume during the bench press exercise? Int $\mathbf{J}$ Environ Res Public Health. 2020;17(2554):1-10.

32. Krzysztofik M, Wilk M. The effects of plyometric conditioning on post-activation bench press performance. J Hum Kinet. 2020;74:7-20.

33. Markovic G, Simek S, Bradic A. Are acute effects of maximal dynamic contractions on upper-body ballistic performance load specific? J Strength Cond Res. 2008;22(6):1811-5.

34. Brandeburg JP. The acute effects of prior dynamic resistance exercise using different loads on subsequent upper-body explosive performance in resistance-trained men. J Strength Cond Res. 2005;19(2):427-32.

35. Esformes JI, Keenan M, Moody J, Bampouras TM. Effects of different types of conditioning contraction on upper body postactivation potentiation. J Strength Cond Res. 2011;25(1):143-8.

36. Montoya BS, Brown LE, Coburn JW, Zinder SM. Effect of warmup with different weighted bats on normal baseball bat velocity. $\mathrm{J}$ Strength Cond Res. 2009;23(5):1566-9.

37. Bellar D, Judge LW, Turk M, Judge M. Efficacy of potentiation of performance through overweight implement throws on male and female collegiate and elite weight throwers. J Strength Cond Res. 2012;26(6):1469-74.

38. Bevan HR, Owen NJ, Cunnigham DJ, Kinglsey MIC, Kilduff LP. Complex training in professional rugby players: influence of recovery time on upper-body power output. J Strength Cond Res. 2009;23(6):1780-5.
39. Bodden D, Suchomel TJ, Lates A, Anagost N, Moran MF, Taber CBT. Acute effects of ballistic and non-ballistic bench press on plyometric push-up performance. Sports. 2019;7(2):47. https:// doi.org/10.3390/sports7020047.

40. Kilduff LP, Bevan HR, Kingsley MIC, Owen NJ, Bennett MA, Bunce PJ, et al. Postactivation potentiation in professional rugby players: optimal recovery. J Strength Cond Res. 2007;21(4):1134-8.

41. Liossis LD, Forsyth J, Liossis G, Tsolakis C. The acute effect of upper-body complex training on power output of martial art athletes as measured by the bench press throw exercise. J Hum Kinet. 2013;39:167-75.

42. Judge LW, Bellar DM, Craig BW, Gilreath EL, Cappos SA, Thrasher AB. Influence of postactivation potentiation on shot put performance of collegiate throwers. J Strength Cond Res. 2016;30(2):438-45.

43. Gilmore SL, Brilla LR, Surprak DN, Chalmers GR, Dahlquist DT. Effect of high-intensity isometric potentiating warm-up on bat velocity. J Strength Cond Res. 2019;33(1):152-8.

44. Hancock AP, Sparks KE, Kullman EL. Postactivation potentiation enhances swim performance in collegiate swimmers. J Strength Cond Res. 2015;29(4):912-7.

45. Barbosa TM, Yam JW, Lum D, Balasekaran G, Marinho DA. Arm-pull thrust in human swimming and the effect of post-activation potentiation. Sci Rep. 2020;10(1):8464.

46. Martínez-Garciá D, Rodríguez-Perea Á, Huerta-Ojeda Á, JerezMayorga D, Aguilar-Martínez D, Chirosa-Rios I, et al. Effects of pre-activation with variable intra-repetition resistance on throwing velocity in female handball players: a methodological proposal. J Hum Kinet. 2021;77(1):235-44.

47. Gelen E, Dede M, Bingul BM, Bulgan C, Aydin M. Acute effects of static stretching, dynamic exercises, and high volume upper extremity plyometric activity on tennis serve performance. J Sports Sci Med. 2012;11(4):600-5.

48. Cuenca-Fernández F, Batalha NM, Ruiz-Navarro JJ, MoralesOrtiz E, López-Contreras G, Arellano R. Post high intensity pullover semi-tethered swimming potentiation in national competitive swimmers. J Sports Med Phys Fitness. 2020;60(12):1526-35.

49. Bliss A, Livingstone H, Tallent J. Field-based and overspeed potentiated warm-ups increase clubhead speed and drive carry distance in skilled collegiate golfers. J Sport Exerc Sci. 2021;5(2):107-13.

50. Feros SA, Hinck K, Dwyer J. The effects of modified-implement warm-ups on cricket pace-bowling skill. Int J Sports Physiol Perform. 2021;28:1-7.

51. Higuchi T, Nagami T, Mizuguchi N, Anderson T. The acute and chronic effects of isometric contraction conditioning on baseball bat velocity. J Strength Cond Res. 2013;27(1):216-22.

52. Williams CC, Gdovin JR, Wilson SJ, Cazas-Moreno VL, Eason JD, Hoke EL, et al. The effects of various weighted implements on baseball swing kinematics in collegiate baseball players. J Strength Cond Res. 2019;33(5):1347-53.

53. Sarramian VG, Turner AN, Greenhalgh AK. Effect of postactivation potentiation on fifty-meter freestyle in national swimmers. J Strength Cond Res. 2015;29(4):1003-9.

54. Smilios I, Sotiropoulos K, Barzouka K, Christou M, Tokmakidis SP. Contrast loading increases upper body power output in junior volleyball athletes. Pediatr Exerc Sci. 2017;29(1):103-8.

55. Asencio P, Sabido R, Garcia-Valverde A, Hernandez-Davo JL. Does handball throwing velocity increase after an eccentric overload-induced postactivation potentiation? Eur J Hum Mov. 2020;44:5-18.

56. Lum D. Effects of various warm-up protocol on special judo fitness test performance. J Strength Cond Res. 2019;33(2):459-65.

57. Aandahl HS, Heimburg V, Vanillaar E. Effect of postactivation potentiation induced by elastic resistance on kinematics and 
performance in a roundhouse kick of trained martial arts practitioners. J Strength Cond Res. 2018;32(4):990-6.

58. Moore LH, Tankovich MJ, Riemann BL, Davies GJ. Kinematic analysis of four plyometric push-up variations. Int J Exerc Sci. 2012;5(4):334-43.

59. Rassier DE, Macintosh BR. Coexistence of potentiation and fatigue in skeletal muscle. Braz J Med Biol Res. 2000;33(5):499-508.
60. Waldron M, Highton J, Gray A. Effects of familiarization on reliability of muscle-activation and gross efficiency in adolescents and adults. Cogent Med. 2016. https://doi.org/10.1080/2331205X. 2016.1237606.

61. Hopkins WG, Schabort EJ, Hawley JA. Reliability of power in physical performance tests. Sports Med. 2001;31(3):211-34.

62. Cuijpers P, Weitz E, Cristea IA, Twisk J. Pre-post effect sizes should be avoided in meta-analyses. Epidemiol Psychiatr Sci. 2017;26(4):364-8. 